\title{
An Effective Reactive Power Compensation Method and a Modern Metaheuristic Algorithm for Loss Reduction in Distribution Power Networks
}

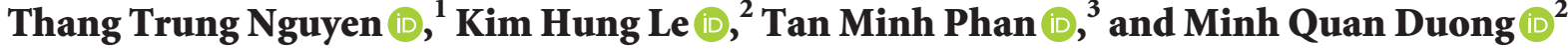 \\ ${ }^{1}$ Power System Optimization Research Group, Faculty of Electrical and Electronics Engineering, Ton Duc Thang University, \\ Ho Chi Minh City 700000, Vietnam \\ ${ }^{2}$ Department of Electrical Engineering, University of Science and Technology-The University of DaNang, \\ Da Nang city 55000, Vietnam \\ ${ }^{3}$ Faculty of Electrical and Electronics Engineering, Ton Duc Thang University, Ho Chi Minh City 700000, Vietnam \\ Correspondence should be addressed to Minh Quan Duong; dmquan@dut.udn.vn
}

Received 17 July 2021; Revised 24 September 2021; Accepted 8 October 2021; Published 26 October 2021

Academic Editor: Mohammad Hassan Khooban

Copyright (C) 2021 Thang Trung Nguyen et al. This is an open access article distributed under the Creative Commons Attribution License, which permits unrestricted use, distribution, and reproduction in any medium, provided the original work is properly cited.

\begin{abstract}
In this paper, a new method of reactive power compensation is proposed for reducing power loss of distribution power networks. The new method is the combination of local compensation at each load and distribution line compensation. In the method, local capacitors at each load are determined to increase power factor of load to an expected value first and then a number of capacitors are placed in distribution lines with two factors, location and capacity by using the three applied methods. Particle swarm optimization (PSO), parasitism predation algorithm (PPA), and tunicate swarm algorithm (TSA) are applied for the proposed method on four distribution systems with $15,33,69$, and 85 buses. The comparisons of results with previous methods indicate that the three applied methods can reach higher loss reduction for all study cases. TSA can reach loss reduction more effectively than others by $19.4 \%, 0.5 \%, 7.9 \%$, and $10.76 \%$ for the four distribution systems, respectively. The most important emphasis is that PSO, which was considered to be of low effectiveness in previous studies, can reach much better loss than approximately all previous methods thank to the proposed compensation method. PSO also reached better loss reduction than others by $18.97 \%, 0.4 \%, 7.73 \%$, and $10.21 \%$, respectively. Consequently, it is recommended that the proposed compensation method is useful for distribution systems in minimizing total power loss and TSA can be selected a new and powerful method for the problem.
\end{abstract}

\section{Introduction}

1.1. Motivation. The analysis of total active power loss and voltage drop in power systems has a very important role in proposing effective methods for optimizing the operation of the power systems, increasing benefit, and stabilizing power networks. Distribution networks directly supply electricity to loads with power much less than power in transmission networks but the total active power loss in the networks is significant due to the use of the low voltage and conductors with high resistance. Both active power flows and reactive power flows in distribution lines cause a high power loss due to the impact of the equation $\left[\left(P^{2}+Q^{2}\right) / U^{2}\right] R$ in which $P$ and
$Q$ are active and reactive power flows, $U$ is the voltage, and $R$ is the resistance of the distribution line. In order to reduce the high power loss, some methods can be used as follows:

(1) Reduce active power flow in the distribution line (i.e., reduce $P$ ). The method can be successful if distributed generation sources like photovoltaic systems, wind turbines, and diesel generator are used.

(2) Reduce reactive power flow in the distribution line (i.e., reduce $Q$ ). In order to reach the reduction of active power flow in lines, distributed generation sources as well as capacitors are placed in the network. 
(3) Increase voltage (i.e., increase $U$ ).

(4) Reduce resistance of the distribution line (i.e., reduce $R)$.

Among the four methods above, the third has an insignificant impact on the loss reduction because the level of voltage improvement cannot be high while the impact of active power and reactive power is very high. The last method seems to be impossible because the reduction of $R$ is equivalent to the replacement of conductors. Conductors with smaller area should be replaced with other ones with higher area. This will cost high amount of money but the benefit is not much. For the first two methods, the power loss can be reduced significantly if active and reactive power flows in the distribution lines are reduced. The reduction is only done by using distributed generation sources such PV systems, wind turbines, diesel generators, and capacitors. The four types of distributed generation sources can be divided into three types: (1) pure active power source, (2) pure reactive power source, and (3) active and reactive power source. Among the three type sources, pure reactive power source, which is capacitors, is the cheapest but its contribution to the loss reduction is significant. So, in this paper we will discuss the impact of capacitors on the loss reduction and then we will propose an effective method of pure reactive power compensation for reducing active power loss in distribution networks.

1.2. Literature Review. In recent decades, many studies have focused on the active power loss reduction by proposing different reactive power compensation methods and different optimization algorithms. About the reactive power compensation methods, authors have proposed two general methods where the first method was to select the compensation location first and then determine the reactive power generation while the second method was to determine compensation location and compensated reactive power simultaneously. The first method can be called two-step method while the second method can be called simultaneous method. In the two-step method, authors could use different methods for selecting the most appropriate locations out of all nodes in the considered distribution network and then authors used one more method to allocate the best power for the capacitors placed at the determined nodes. Normally, the two-step methods were used for weak methods that were employed to calculate the generation of capacitors. On the contrary, the simultaneous methods were applied for more powerful search algorithms, which could find location and size of capacitors simultaneously. A two-step method was employed for the reduction of the active power loss in a 15node distribution system and a 33-node distribution system [1]. An iterative algorithm was run to determine the most appropriate compensation nodes in the first step while a power loss minimization equation with respect to the current of the capacitor was solved for reaching the size of capacitors at each predetermined location. The method could reduce power loss more effectively than base network without capacitors; however, the method had to suffer from the disadvantage of the first step. In fact, for the case of placing a high number of capacitors, the method was time consuming because one by one capacitor was run to get the compensated location until the location selection of the last capacitor was accomplished. The main disadvantage of the method leads to the main shortcoming that it cannot be highly effective for large distribution systems with too many nodes and feeders. Two-step methods were proposed in the study [2] by using a two-loop algorithm, called outer layer and inner layer. Outer layer was activated to find the optimal location of added switchable shunt capacitors in the first loop. Then, inner layer was run in the second loop to determine the size of each added capacitor. The inner layer was the operation of metaheuristic algorithms. The methods were run on two systems with 33 and 69 nodes and compared to a few methods for conclusion of performance. Among the number of proposed approaches in [2], only approach based on genetic algorithm could reach better losses than other previous approaches. The two-step method [1] continued to be applied in [3] for determining location and size of distributed generators instead of capacitors and promising results could be achieved since the total power loss was less than the case without capacitor placement. However, the successful and effective applicability of the method was not proved for other larger scale systems with 69 and 85 nodes. On the contrary to the studies in $[1,2]$, the two-step method in [4] has applied fuzzy system (FS) and loss reduction factor (LRF) in the first step for getting the most suitable compensated nodes. The two-step method was applied widely and successfully for different test cases from small systems to large systems thanks to the combination of FS and LRF, which was not influenced by the system scale. Actually, the method has reached less active power loss than base network and other applied methods for a 69-node distribution system. The main shortcoming of the method was long simulation time due to the combination of FS and the calculation of LRF. In [5], only LRF was employed in the first step to evaluate the operation effectiveness of each node in distribution networks and then compensated nodes were selected based on the value of LRF. In the second step, an improved particle swarm optimization algorithm (WCPSO) using weight and constriction factors was applied to calculate reactive power generation of each capacitor at the predetermined node. The combination of LRF and WCPSO has formed an effective two-step method that has successfully dealt with five systems consisting of 10, 15, 34, 69, and 85 buses in reaching voltage improvement. The method in [5] has been compared to another two-step method in [1] in terms of voltage profile and the conclusion was that it was much superior to the two-step method in [1]. However, the comparison was not reasonable because the objective in [1] was the minimization of power loss but that in [5] was the voltage profile optimization. Consequently, the real effectiveness of the combination of LRF and WCPSO was still a question. Another two-step method has been proposed in [6] by applying fuzzy method in the first step to select compensation nodes and applying WCPSO in the second step to calculate the reactive power of capacitors at the determined nodes. The two-step method successfully solved 
a biobjective problem with loss reduction and voltage improvement for the 69-node distribution system. Both loss and voltage have been demonstrated to be much better than those in the base network without capacitor placement but there was no further comparison with other applied methods and previous methods. In [7], the fuzzy method in [6] has been replicated in the first step and an improved particle swarm optimization (IPSO) has been proposed in the second step for capacitor size determination. The study has applied another two-step method based on the fuzzy method and conventional PSO to compare the power loss and voltage profile with the proposed method based on fuzzy method and IPSO for the 69-node distribution network. The results of simulation have indicated that IPSO could find capacity more effectively than PSO with the same settings of control parameters. The discussion on the studies [1-7] has indicated that the two-step methods were not strong in placing capacitors for minimizing total active power loss in distribution lines. The main shortcomings of the methods are either the methods applied in the first step for finding compensation nodes or the methods applied in the second step for determining the size of capacitors. In order to improve the performance of two-step methods, other more effective metaheuristics have been used for the step of finding the size of capacitors in recent years such as improved harmony algorithm (IHA), ant colony algorithm (ACA), and modified particle swarm optimization (MPSO). The new two-step methods are the power loss index and improved harmony algorithm-based method (PLIIHA) [8], the loss sensitivity factor and ant colony algorithm-based method (LSFACA) [9], and the power loss analysis and MPSO based method (PLA-MPSO) [10]. Based on more effective metaheuristic methods, these methods in [2-4] could be capable of solving larger scale systems with better obtained results. In [10], a network analysis method in [11] has been applied to calculate the power loss function and then MPSO has been applied for two study cases. In the first study case, MPSO was used to find locations and then power loss function was used to calculate the size of capacitors. In the second study case, MPSO was used to determine both location and size of capacitors. The methods in the two study cases are named hybrid MPSO and network analysis method (HM) and MPSO. As a result, MPSO was more useful than HM for 33- and 69-node distribution systems. However, the two methods have not been compared to other methods excluding analysis method (AM) in [11]. Network configuration analysis-based methods (NCAB) have become more promising and the applications of metaheuristics in the methods have not been necessary [11-15]. On the contrary to metaheuristic algorithms, all NCAB methods have not applied randomization in solution update process and they have reached the same optimal solution for different trial runs. These methods could be applied for larger scale systems up to 69 and 85 nodes, and the results were effective; however, these methods had to suffer from a main drawback, which was the difficult applicability. In fact, these methods were based on mathematical analysis on the current data of networks such as resistance and reactance of lines and active and reactive power of loads. So, as the data are changed, the methods had to be reimplemented. In other words, these methods could not be applied for general systems and the implementation of the methods for different configurations of network has to be different.

On the contrary to these two-step methods and NCAB methods, metaheuristics could be applied widely for systems without using the two separate steps as the two-step methods as well as without using the network configuration analysis as NCAB methods. These methods use two main control variables including location and generation of capacitors. The total number of main control variables $\left(N_{\mathrm{mcv}}\right)$ is directly proportional to the number of placed capacitors $\left(N_{\text {cap }}\right)$. If a distribution network is placed with $N_{\text {cap }}$ capacitors, $N_{\text {mcv }}$ will be equal to $\left(2 \times N_{\text {mcv }}\right)$ in which $N_{\text {mcv }}$ values are locations and $N_{\text {mcv }}$ values are sizes. After determining the site and the generation of capacitors, a load power flow method is applied to calculate current flows in branches. Then voltage and power loss can be determined by using voltage drop calculation formula and power loss calculation formula. Basically, two popular power flow methods that are employed for distribution networks are Newton-Raphson method and forward/backward technique. In [16], fifteen differently modified versions of conventional PSO were applied for the selection of compensation nodes and size of capacitors of a 10 -node distribution system. The best modified particle swarm optimization (BMPSO) among the fifteen applied ones was compared to tabu search algorithm (TSA) and conventional genetic algorithm (GA) in terms of power loss and the conclusion was that BMPSO was more powerful than TSA and GA. Although BMPSO was the best among fifteen PSO methods and two other methods, its real effectiveness for the problem was not demonstrated persuasively. In fact, only a very simple system with 10 nodes and one main feeder was utilized as a study case, and the comparison was implemented only among PSO methods and few other methods.

GA has been also applied for the problem in many studies so far [17-23]. The application of GA for the problem was wide from small scale system with 15 nodes to largescale system with 69 nodes even for real distribution networks in Taiwan and Iran. But the most important disadvantage of the studies was the poor demonstration for the effectiveness [24]. It seemed that these studies only focused on the power loss reduction as compared to initial network without any compensation devices rather than showing the effectiveness of the applied methods. Consequently, the comparisons with other methods have not been concerned in the studies. Furthermore, the data of real distribution networks in Taiwan and Iran were not shown in the studies for checking and replicating the published results. This is a reason that the distribution networks of the two countries cannot be run by other methods. In addition, other metaheuristics were also applied for the capacitor placement in distribution systems such as bacterial foraging algorithm (BFA) [25, 26], gravitational search algorithm (GSA) [27], teaching and learning based algorithm (TLBA) [28], improved differential evolution (IDE) [29], flower pollination algorithm (FPA) [30, 31], whale optimization algorithm (WOA) [32], cuckoo search algorithm (CSA) [33], moth 
swarm algorithm (MSA) [34], and the hybrid GSA and improved particle swarm optimization method (HGSAIPSO) [35]. These metaheuristics' performance has been shown effectively by comparing them with other methods and original network without the placement of capacitors. Power loss reduction was more significant than other twostep methods and the trial distribution networks were more plentiful. Nevertheless, all the studies have only focused on the power loss reduction where the computation complexity and computation speed have not been concerned as important issues. It may be that these authors have considered the capacitor placement problem in distribution networks was offline problem and computation speed was not an important criterion. However, all electric loads in distribution networks can change consumption power continuously, leading to the change of current in conductors and load voltage. So, computation speed of applied methods has a very important role in selecting operation modes that can help systems to work effectively and stably. Derived from the issue, in this paper, we concern the computation speed as an important criterion during the implementation of applied methods for determining capacitors in distribution system in addition to the power loss reduction.

1.3. Novelties and Contributions. In this study, we apply the policy of power companies in increasing the power factor of loads. The fact that reactive loads in distribution power grids use high reactive power and power source must be the high demand of reactive power to loads. This leads to high reactive power flows in distribution lines, causing high active power loss. After applying reactive power compensation policy of the power companies for increasing load power factor, some other capacitors are placed in distribution lines to reduce total active power loss and increase voltage of loads. In the first step, given power factor of each load node is predetermined and then capacitor at the load node is calculated based on the known power factor, active power, and reactive power of the load. In the second step, the total compensation power of all capacitors at electric loads is determined. In the third step, total power of capacitors placed on distribution lines is calculated. In the last step, the number of capacitor banks and the power of the capacitor banks are determined by using metaheuristic algorithms. Thus, three metaheuristic algorithms including particle swarm optimization (PSO) [36], parasitism predation algorithm (PPA) [37], and tunicate swarm algorithm (TSA) [38] are applied to do the last step and found power losses are compared to previous methods. In summary, the novelties of the paper are as follows:

(1) Propose a new reactive power compensation method where the reactive power compensation policy of power companies is implemented in the first step. This application is realistic and effective for distribution networks.

(2) Apply new metaheuristic algorithms including PPA and TSA to test real performance and select the best one for the problem of placing capacitors in distribution networks.

Thanks to the novelties above, the main contributions of the paper are as follows:

(1) Reduce the total power loss in distribution system effectively.

(2) Introduce new metaheuristics that readers can use for other optimization problems in power systems or in other fields.

(3) Reach the best performance for TSA for loss reduction by selecting the most appropriate parameters.

(4) Provide an effective reactive power compensation method to distribution network. By using the proposed method, a low performance method like conventional PSO also reaches better results than almost all compared methods.

In addition to the introduction, the paper also contains some other parts as follows. The concerned objective and constraints are shown in Section 2. Section 3 shows the search process of TSA. In Section 4, a new method of reactive power compensation is presented in detail. In Section 5, numerical results are shown and discussed. Finally, conclusions are summarized in Section 6.

\section{Problem Formulation}

2.1. The Concerned Objective. Distribution networks consist of many distribution lines where conductors with small area and high resistance are in charge of transferring electricity to loads. Although power flows in the lines are not high and much less than that in transmission lines, their low voltage is a main cause of leading to high total active power loss. Consequently, the total active power loss (TAPL) in all distribution lines is highly concerned in distribution network operation and it must be reduced as much as possible. The objective is mathematically described as follows:

$$
\text { Minimize TAPL }=\sum_{l=1}^{\mathrm{Ndl}} \Delta P_{l} \text {, }
$$

where

$$
\Delta P_{l}=3 I_{l}^{2} R_{l}
$$

where $\Delta P_{l}$ is the active power loss in the lth distribution line due to the impact of the resistance $R_{l}$ of the $l$ th distribution line and the current $I_{l}$ flowing in the $l$ th distribution line. $I_{l}$ is calculated by running forward backward sweep method (FBSM) [39] and it is also used to calculate the voltage drop on the lth distribution line as follows:

$$
\Delta U_{l}=\sqrt{3} I_{l} Z_{l}
$$

Then the voltage drop is applied to calculate voltage at the receiving node of the $l$ th distribution line. 


\subsection{Constraints}

2.2.1. Current Limit. The main parameter of distribution line regarding its operation status is temperature. Due to the impact of environment temperature and current on the temperature of line, current flowing lines have to be supervised and constrained by

$$
I_{l} \leq I_{l}^{\max } ; \quad l=1, \ldots, N l,
$$

where $I_{l}^{\max }$ is the maximum limit of current flowing in the $l$ th distribution line.

2.2.2. Voltage Limit. Voltage is a power quality factor playing an important role in evaluating stable and effective operation of distribution networks. All loads have to work within an allowable voltage range between the lower limit and the upper limit as the following expression:

$$
U^{\min } \leq U_{k} \leq U^{\max } ; \quad k=2, \ldots, N n .
$$

2.2.3. Limit of Capacitors. The reactive power of all capacitors placed in a system must be limited as the following inequality:

$$
\sum_{c=1}^{N c a p} Q_{\text {capc }} \leq Q_{\text {cap }}^{\max }
$$

where $Q_{\text {capc }}$ is the generation of the $c$ th capacitor; and $Q_{\text {cap }}^{\max }$ is the maximum generation of all capacitors. Normally, the maximum generation of all capacitors is selected not to be higher than the total reactive power of all loads in the system. If there are no generation limits of all capacitors, the maximum generation can be infinite; however, a very high value of the maximum generation is only useful for voltage improvement but it may be not very good for the purpose of power loss reduction. Consequently, the maximum generation of all capacitors can be selected as follows:

$$
Q_{\text {cap }}^{\max } \leq \sum_{k=1}^{N n} Q_{\text {Loadk }} .
$$

\section{Tunicate Swarm Algorithm (TSA)}

In the paper, three metaheuristics are applied, that is, PSO, PPA, and TSA. However, PSO is one of the earliest methods that has been used in many studies and PPA is not more effective than TSA. So, TSA is selected to be a potential method for the concerned problem. The main configuration of the potential method is presented in detail as follows.

3.1. Initialization of Population. Like most metaheuristic methods, TSA also produces the initial population in the first step of the whole search procedure. Each solution is represented by $S_{x}$ (where $x=1, \ldots$, Npo and Npo is population size) and initialized as follows:

$$
S_{x}=S^{\min }+\varepsilon_{1}\left(S^{\max }-S^{\min }\right) .
$$

In the equation, $S^{\min }$ and $S^{\max }$ are the lower bound solution and upper bound solution. The two solutions contain the minimum value and maximum value of all control variables, respectively. After the initialization, each solution $S_{x}$ is evaluated by calculating fitness function $\left(\mathrm{FF}_{x}\right)$ and $S_{x}$ with the lowest value of $\mathrm{FF}_{x}$ becomes the best solution $S_{G \text { best. }}$.

3.2. The Update for the First Solution. In the second step of TSA, the first solution is updated first by using the following equation:

$$
S_{1}^{\text {new }}= \begin{cases}S_{G \text { best }}-A \times \Delta S_{1}, & \text { if } r d_{1}<0.5, \\ S_{G \text { best }}+A \times \Delta S_{1}, & \text { otherwise, }\end{cases}
$$

where $\Delta S_{1}$ and $A$ are calculated by

$$
\begin{aligned}
\Delta S_{1} & =\left|S_{G \text { best }}-\varepsilon_{2} \times S_{1}\right|, \\
A & =\frac{B}{C},
\end{aligned}
$$

where

$$
\begin{aligned}
B & =\gamma_{1}+\gamma_{2}-2 \gamma_{3}, \\
C & =R d_{L}+\varepsilon_{3}\left(R d_{U}-R d_{L}\right) .
\end{aligned}
$$

$B$ and $C$ in equations (11) and (12) are obtained by using random parameters including $\gamma_{1}, \gamma_{2}, \gamma_{3}$, and $\varepsilon_{3}$. The four parameters are produced randomly within 0 and 1 while $R d_{L}$ and $R d_{U}$ in formula (12) are the minimum and maximum values of $C$, which are, respectively, selected to be 1 and 4 by experiment [38]. Clearly, $B$ has a value range from -2 to 2 and the range of $C$ is from 1 to 4 . If $B$ has a high value and $C$ has a small value, $A$ will become larger and vice versa. It is noted that $A$ is used in equation (9) to obtain the result of $\left(A \times \Delta S_{1}\right)$, which is a jumping step to update the first new solution around the best solution $S_{G \text { best. }}$. If $A$ has a small value, the new solution $S_{1}^{\text {new }}$ will be close to $S_{G \text { best }}$ and if $A$ has a high value, the new solution $S_{1}^{\text {new }}$ is far from $S_{\text {Gbest }}$. As demonstrated in [38], the value of 1 for $R d_{L}$ and the value of 4 for $R d_{U}$ were proved to be effective for benchmark functions. However, optimal solutions of benchmark functions in [38] are approximately comprised of zero values and these benchmark functions are totally different from the reactive power compensation problem. By experiment, we use $C$ in a large range from 1 to 10 to cause a smaller value for $A$ and it is useful to reach a more promising new solution nearby the best solution $S_{G \text { best }}$. So, $R d_{L}$ and $R d_{U}$ are set to 1 and 10 to get a high performance for TSA for the reactive power compensation problem in distribution systems and these set values can enable TSA to find better results than 1 and 4 as suggested in [38]. By experiment, we have tested two options for $R d_{L}$ and $R d_{U}$ including 1 and 4 (as suggested in [38]) and 1 and 10 (proposed in the study) to reach the results of TSA for the first two systems with 15 and 33 buses. The final results for the two systems indicated that TSA had a 
better performance when applying the proposed values of 1 and 10. And then we suggested using the setting for two remaining systems with 69 and 85 nodes.

3.3. The Update for Other Solutions. In the third step, the second solution to the final solution are newly updated one by one. The update process is implemented from the front solution to the behind solution until the update for final solution is finished. The update can be proceeded by applying the three following formulas:

$$
\begin{aligned}
\Delta S_{x} & =\left|S_{G \text { best }}-\varepsilon_{4} \times S_{x}\right|, \\
S_{x}^{\prime} & = \begin{cases}S_{G \text { best }}-A \times \Delta S_{x}, & \text { if } r d_{x}<0.5, \\
S_{G \text { best }}+A \times \Delta S_{x}, & \text { otherwise, }\end{cases} \\
S_{x}^{\text {new }} & =\frac{S_{x}^{\prime}+S_{x-1}^{\text {new }}}{2+\varepsilon_{5}} .
\end{aligned}
$$

3.4. Verification and Correction for the New Solutions. After producing a new solution, an important step that cannot be missed in metaheuristic algorithms is to check and correct the violation of the new solution. If the new solution is beyond the upper bound solution $S^{\max }$ and the lower bound solution $S^{\mathrm{min}}$, it is fixed as shown in the following formula:

$$
S_{x}^{\text {new }}= \begin{cases}S^{\mathrm{min}}, & \text { if } S_{x}^{\text {new }}<S^{\mathrm{min}}, \\ S^{\mathrm{max}}, & \text { if } S_{x}^{\text {new }}>S^{\mathrm{min}}\end{cases}
$$

3.5. Selecting and Retaining High-Quality Solutions. The quality of all new solutions has to be evaluated by calculating fitness function. If $\mathrm{FF}_{x}$ is the fitness function of the old solution $S_{x}, \mathrm{FF}_{x}^{\text {new }}$ is the fitness function of the new solution $S_{x}^{\text {new }}$. At each position $x$, either $S_{x}$ or $S_{x}^{\text {new }}$ is retained and assigned to ${ }_{S}^{x}$. The expression is called selection mechanism and simply formulated by the following equations:

$$
\begin{aligned}
S_{x} & = \begin{cases}S_{x}^{\text {new }}, & \text { if } \mathrm{FF}_{x}^{\text {new }} \leq \mathrm{FF}_{x}, \\
S_{x}, & \text { else, }\end{cases} \\
\mathrm{FF}_{x} & = \begin{cases}\mathrm{FF}_{x}^{\text {new }}, & \text { if } \mathrm{FF}_{x}^{\text {new }} \leq \mathrm{FF}_{x}, \\
\mathrm{FF}_{x}, & \text { else. }\end{cases}
\end{aligned}
$$

3.6. The Whole Search Procedure of TSA. The whole computation process of TSA for solving a general optimization problem can be summarized as the following steps and described in the flowchart of Figure 1.

Step 1: select values for population Npo and iterations Niter

Step 2: define the lower bound solution $S^{\min }$ and the upper bound solution $S^{\max }$
Step 3: randomly generate the initial population by using equation (8)

Step 4: calculate fitness function of each solution

(i) Select the best solution among all solutions

(ii) Set the current iteration to $1($ Citer $=1)$

Step 5: apply equation (9) to update the first new solution

Step 6: apply equation (16) to check and correct the first new solution

Step 7: apply the pseudo code below for updating the second new solution to the last new solution

For $x=2: \mathrm{Npo}$

Calculate $\Delta S_{x}$ and $S_{x}^{\prime}$ by using equations (13) and (14)

Update new solution by using equation (15)

Check and correct the newly updated solution by using equation (16)

end

Step 8: calculate fitness function of the new solutions Step 9: apply equations (17) and (18) to retain promising solutions

Step 10: select the best solution among all solutions

Step 11: if Citer $=$ Niter, stop proceeding TSA. Otherwise, set Citer $=$ Citer +1 and back to Step 5 .

\section{The Implementation of Capacitor Placement in Distribution Systems}

4.1. Calculation of Total Compensation Capacity at Loads. The power factor of each load can be calculated by using active and reactive power as follows:

$$
P F_{k}=\frac{P_{k}}{\sqrt{P_{k}^{2}+Q_{k}^{2}}} ; \quad k=2, \ldots, N n .
$$

Then compensation at the load $k$ is calculated by

$$
Q_{\text {cap }, k}=P_{k}\left(\operatorname{Tan} \varphi_{k}-\operatorname{Tan}\left(\arccos \varphi^{\text {Request }}\right)\right) ; \quad k=2, \ldots, N n \text {, }
$$

where

$$
\operatorname{Tan} \varphi_{k}=\frac{Q_{k}}{P_{k}}
$$

and $\cos \varphi^{\text {Request }}$ is the expected power factor required by the power companies and it is given by the power companies. In case that load is compensated by using smaller value than $\cos \varphi^{\text {Request }}$, customer must be penalized. by

The total compensation capacity at loads is determined

$$
Q_{\text {total }}=\sum_{k=2}^{N n} Q_{\text {cap }, k}
$$

4.2. Calculation of Total Compensation Capacity at Nodes in Distribution Lines. Then the total compensation capacity in distribution system is calculated by 


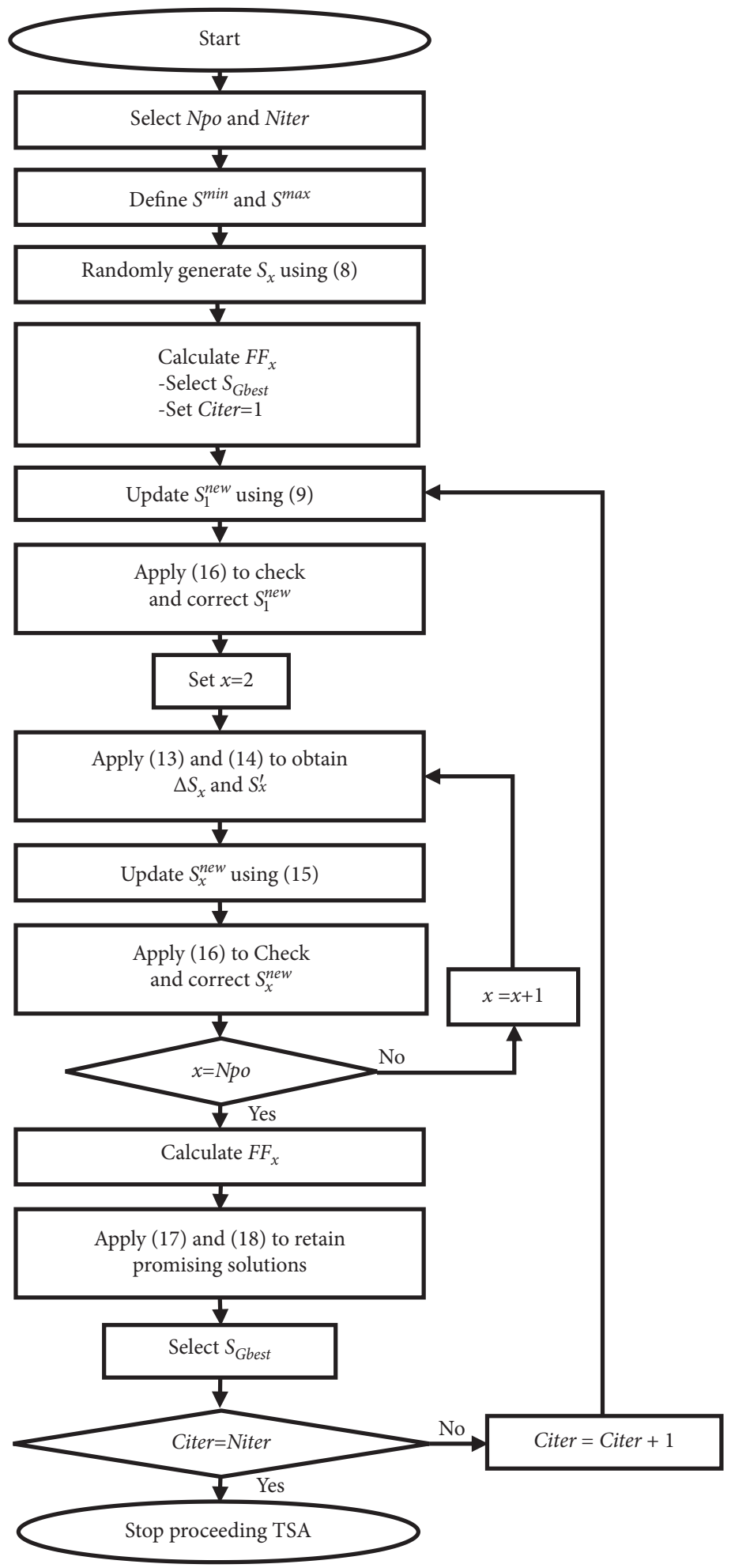

FIGURE 1: Flowchart of using TSA for solving a typical optimization problem.

$$
\sum_{c=1}^{N \text { cap }} Q_{\text {capc }} \leq Q_{\text {cap }}^{\text {max }}-Q_{\text {total }} .
$$

In summary, we implement compensation of reactive power twice. In the first stage, we compensate reactive power at loads by using equation (20) to increase their power factor and then we calculated the total compensation power $Q_{\text {total }}$ at loads by using equation (22). In the second stage, the upper limit of total compensation capacity at nodes on distribution lines is obtained by using equation (23). In equation (23), the total compensation capacity at nodes on 
distribution lines is $\sum_{c=1}^{N c a p} Q_{\text {capc }}$ and the total compensation capacity at loads is $Q_{\text {total }}$. In previous studies, the compensation of reactive power at loads was not performed. Thus, $Q_{\text {total }}$ at loads was not considered and the constraint of $\left(\sum_{c=1}^{N c a p} Q_{\text {capc }} \leq Q_{\text {cap }}^{\max }\right)$ was used instead of the constraint (23).

\subsection{The Implementation of TSA for Capacitor Placement in Distribution Systems}

4.3.1. The Selection of Control Variables. TSA is applied to find the compensated nodes in distribution systems and the compensation capacity of the selected nodes. So, the two main control variables are compensation nodes and compensation capacity, which are represented by $C N_{c}$ and $Q_{\text {cap } c}$ $\left(c=1, \ldots, N\right.$ cap). Among the two variables, $C N_{c}$ is within 2 to $N n$ while $Q_{\text {capc }}$ is from 0 to less than $\left(Q_{\text {cap }}^{\max }-Q_{\text {total }}\right)$. Furthermore, all capacitors must satisfy the constraint (23) above. The smallest values of $C N_{c}$ and $Q_{\text {capc }}$ are called the minimum boundary solution and the highest values of the two variables are called the maximum boundary solution. The boundary solutions are defined as follows:

$$
\begin{aligned}
& S^{\min }=\left[C N_{c}^{\min }, Q_{\text {capc }}^{\min }\right] ; \quad c=1, \ldots, N \text { cap }, \\
& S^{\max }=\left[C N_{c}^{\max }, Q_{\text {cap } c}^{\max }\right] ; \quad c=1, \ldots, N \text { cap }
\end{aligned}
$$

where $C N_{c}^{\min }$ and $C N_{c}^{\max }$ are, respectively, equal to 2 and $N n$; and $Q_{\text {capc }}^{\min }$ and $Q_{\text {capc }}^{\max }$ are, respectively, equal to 0 and $\left(Q_{\text {cap }}^{\max }-Q_{\text {total }}\right)$.

4.3.2. Power Flow Method. After determining the compensation node and the compensation capacity at each compensated node, FBSM [39] is applied to calculate the current flowing in all distribution lines. As a result, voltage of each node and power loss in each distribution line are calculated.

4.3.3. Fitness Function. Fitness function of each solution in the problem is comprised of total power loss and the total of all penalty terms as shown in the following formula:

$$
\mathrm{FF}_{x}=\mathrm{TAPL}+K_{1} \sum_{l=1}^{N l}\left(I_{l}-I_{l}^{\lim }\right)^{2}+K_{2} \sum_{k=2}^{N n}\left(U_{k}-U_{k}^{\lim }\right)^{2}+K_{3}\left(\sum_{c=1}^{N \text { cap }} Q_{\text {capc }}-Q_{\text {cap }}^{\lim }\right)^{2}
$$

In the function above, $K_{1}, K_{2}$, and $K_{3}$ are penalty parameters. These penalty factors are used to make penalty terms bigger in the fitness function. By experiment, we set $10^{3}$ to the three penalty factors $K_{1}, K_{2}$, and $K_{3}$ for all study cases. $I_{l}^{\lim }, U_{k}^{\lim }$, and $Q_{\text {cap }}^{\lim }$ are the limits for the current of the $l$ th line, the voltage of the $k$ th node, and the total generation of capacitors. The three limits are calculated by

$$
\begin{aligned}
I_{l}^{\lim } & = \begin{cases}I_{l}, & \text { if } I_{l} \leq I_{l}^{\max }, \\
I_{l}^{\max }, & \text { else, }\end{cases} \\
U_{k}^{\lim } & = \begin{cases}U_{k}, & \text { if } U_{k}^{\min } \leq U_{k} \leq U_{k}^{\max }, \\
U_{k}^{\max }, & \text { if } U_{k}>U_{k}^{\max }, \\
U_{k}^{\min }, & \text { if } U_{k}<U_{k}^{\min },\end{cases} \\
Q_{\text {cap }}^{\lim } & = \begin{cases}Q_{\text {cap }}^{\max }-Q_{\text {total }}, & \text { if } \sum_{c=1}^{N c a p} Q_{\text {capc }}>Q_{\text {cap }}^{\max }-Q_{\text {total }}, \\
\sum_{c=1}^{N \text { cap }} Q_{\text {cap }}, & \text { else. }\end{cases}
\end{aligned}
$$

4.3.4. Computation Termination Condition. TSA is also a metaheuristic algorithm based on population. So, its search process is implemented until the current iteration is equal to the predetermined maximum iteration.
4.3.5. The Whole Search Procedure of TSA for Reactive Power Compensation in Distribution Networks. The whole computation process of TSA for reactive power compensation in distribution networks can be summarized in the following steps:

Step 1: set value to expected power factor ( $\left.\cos \varphi^{\text {Request }}\right)$ Step 2: calculate $Q_{\text {cap }, k}$ using equation (20)

Step 3: calculate $Q_{\text {total }}$ using equation (22)

Step 4: select values for population Npo and iterations Niter

Step 5: define $S^{\min }$ and $S^{\max }$ using equations (24) and (25)

Step 6: randomly generate $S_{x}(x=1, \ldots$, Npo $)$ within $S^{\min }$ and $S^{\max }$

Step 7: run FBSM to reach $I_{l}$ and $U_{k}$

Step 8: calculate TAPL using equation (1)

Step 9: calculate fitness function $\mathrm{FF}_{x}$ using equations (26)-(29)

(i) Select the best solution $S_{G \text { best }}$ with the best fitness value

(ii) Set Citer $=1$

Step 10: apply equation (9) to update the first new solution

Step 11: apply equation (16) to check and correct the first new solution 
Step 12: apply the pseudo code below for updating the second new solution to the last new solution

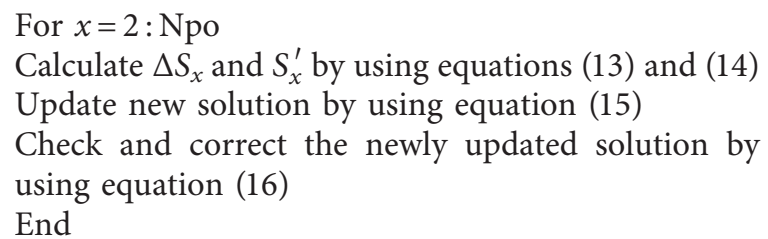

Step 13: run FBSM to reach $I_{l}$ and $U_{k}$

Step 14: calculate TAPL using equation (1)

Step 15: Calculate fitness function $\mathrm{FF}_{x}^{\text {new }}$ using equations (26)-(29)

Step 16: apply equations (17) and (18) to retain promising solutions

Step 17: select the best solution $S_{\text {Gbest }}$

Step 18: if Citer $=$ Niter, stop proceeding TSA. Otherwise, set Citer $=$ Citer +1 and back to Step 10 .

\section{Numerical Results}

In this section, four radial distribution systems with 15,33 , 69 , and 85 buses are considered for capacitor placement. In the first stage, reactive power compensation at each load in the systems is implemented for increasing the power factor into 0.9. In the second stage, metaheuristic methods are employed to determine the location and size of additional capacitors at nodes in distribution lines. In the second stage, three metaheuristic algorithms including PSO, PPA, and TSA have been implemented to determine the location and size of additional capacitors at nodes in distribution lines. Basically, the metaheuristic methods, which are based on randomization in the new solutions update process, search optimal solutions in a predetermined solution space and find out different solutions for different trial runs. Even quality of solutions among the independent runs is totally different, some run with high quality solutions, some run with low quality solutions, and for worse cases, some run with invalid solutions that cannot satisfy all constraints of the problem. To deal with the issue, the invalid solutions and the unsuccessful runs are eliminated by implementing other successful runs to reach valid solutions satisfying all constraints. So, for each study case fifty successful runs are implemented to reach fifty valid solutions and the fifty runs are summarized in terms of the best power loss, mean power loss, worst power loss, and the standard deviation (SD). In tables, we have reported the four values to compare the performance of the three applied methods.

All study cases are implemented by coding the applied methods in Matlab program language and on a Personal Computer with the processor of $2.4 \mathrm{GHz}$ and a RAM of 2.4 GB.

5.1. Comparison and Discussion on Results Obtained for the IEEE 15-Bus Distribution System. In this section, a test system with 15 buses, called IEEE-15 bus distribution system [34], is employed to place capacitors. In the first stage, power factor at each load bus is improved to 0.9 by compensating reactive power factor. The compensation capacity at each load bus is presented in Figure 2. The total capacity of all local capacitors is $657.16 \mathrm{kVAr}$. Then, three methods including PSO, PPA, and TSA are applied to place capacitors in the distribution lines. There are two study cases for the system in which two capacitors are placed in Case 1 and three capacitors are placed in Case 2. The comparison and discussion are as follows.

5.1.1. Case 1: Two Capacitors. To allocate the site and generation of the two capacitors, three methods are run by setting the same control parameters, namely, 10 for Npo and 30 for Niter. The three methods have the same feature with one stage only for updating new solutions. So, the setting is selected to keep fair comparison in implementation. The results in terms of the best power loss, mean power loss, worst power loss, and the standard deviation (SD) are summarized in Table 1. The table can show the best performance of TSA among three applied methods because it can get the lowest values for the best cost and the mean cost, $29.6611 \mathrm{~kW}$ and $30.2492 \mathrm{~kW}$. Although the worst loss and SD of TSA are just the second best result behind PSO with the best values for the worst loss and SD, TSA is still the best one among the three methods since the best optimal solution is the top comparison criteria for the powerful search of method while the mean solution (which is reflected via the mean loss) can indicate the stability of all successful runs. Clearly, TSA is the most powerful and stable search method. The statement can be confirmed by using Figures $3-5$. A very fast search procedure of TSA can be seen in Figure 3 since at the third iteration, TSA can find much better solution than PSO and PPA. As considering the whole search of 50 runs, Figure 4 reveals that, at the eighth iteration, TSA can find much better mean solution than PSO and PPA. Figure 5 can provide the exact number of solutions that TSA can reach better loss than PSO and PPA. About twenty and fifteen better solutions than the best solution of PSO and TSA for 50 runs can be counted in Figure 5. In summary, TSA is much superior to PSO and TSA for Case 1 of the IEEE 15-bus distribution system.

The comparisons of results from the three applied methods and previous methods are shown in Table 2. The power loss of TSA is the lowest and less than two-step method [1] by $2.94 \mathrm{~kW}$, WCPSO [5] by $2.04 \mathrm{~kW}$, and LSFACA [9] by $7.15 \mathrm{~kW}$. The saving loss is, respectively, equivalent to $9.02 \%, 9.3 \%$, and $19.4 \%$ of the loss from twostep method, WCPSO, and LSFACA. In addition, two other applied methods, PSO and PPA, also reach better results than other methods. PSO and PPA can reach less loss than two-step method, WCPSO, and LSFACA by $\{2.772 \mathrm{~kW}$, $2.872 \mathrm{~kW}$, and $6.982 \mathrm{~kW}\}$ and $\{2.864 \mathrm{~kW}, 2.964 \mathrm{~kW}$, and $7.074 \mathrm{~kW}\}$, which are corresponding to $\{8.5 \%, 8.78 \%$, and $18.97 \%\}$ and $\{8.79 \%, 9.06 \%$, and $19.22 \%\}$ of the total power loss from them. Clearly, TSA can reach the most appropriate capacitor placement. The three applied methods are run by setting 10 and 30 to Npo and Niter while other methods have not been shown in studies. The total reactive 


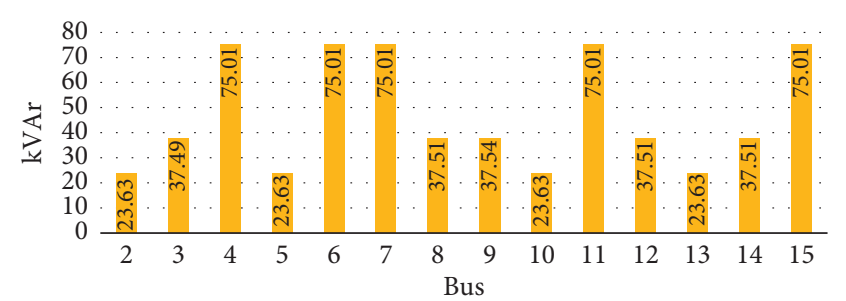

FIgURe 2: Compensation capacity at loads in the IEEE 15-bus distribution system.

power compensated in the system is, respectively, 1193, $1192,1040,1054,1024$, and $1080 \mathrm{kVAr}$ by two-step method, WCPSO, LSFACA, PSO, PPA, and TSA. The comparison indicates that the compensation capacity is not the same for all methods and even for three applied methods. In fact, previous methods have been applied without the limit of total capacity from two capacitors. So, in the study, we also do not constrain the total capacity; however, that from the three applied methods is not higher than that of other ones although the three methods have used two compensation stages in which $527 \mathrm{kVAr}$ is from the first compensation stage and another value is from the two capacitors in the distribution lines. For instance, the generation of two capacitors of PSA, PPA, and TSA is 527, 497 , and $553 \mathrm{kVAr}$. The saving loss and loss improvement percentage indicate that TSA is the best method and the two compensation stages applied for PSO, PPA, and TSA are much more effective than one compensation stage in previous studies.

5.1.2. Case 2: Three Capacitors. In this case, three capacitors are placed in the IEEE 15-bus distribution system by setting 10 and 30 to Npo and Niter for PSO, PPA, and TSA. Table 3 summarizes the results from the three methods for comparisons. All the lowest values regarding power loss and standard deviation provide good evidence to conclude TSA wins PSO and PPA. The feature of search process shown in Figures 6 and 7 can show the superiority of TSA over PSO and PPA since the best run of TSA at the thirteenth iteration can find much better solution than PSO and PPA at the final iteration. The mean characteristic of 50 runs in Figure 7 shows TSA is much effective than PSO and PPA from the 18th iteration. The loss values from 50 runs in Figure 8 provide the most stable search of TSA once many red points have lower loss than the best point of PSO and PPA. Furthermore, the deviation of loss between different runs of TSA is very low while that of PSO and PPA is much higher. Consequently, TSA continues to be the best one among three applied methods for the IEEE 15-bus distribution system.

Comparisons $\mathrm{f}$ or the three applied methods and PLIIHA [8] are shown in Table 4. The loss of TSA is the lowest and less than that of PLIIHA by $1.92 \mathrm{~kW}$ corresponding to $6.2 \%$ of total loss from PLIIHA. Similarly, PSO and PPA can reach less loss than PLIIHA by 1.32 and $1.47 \mathrm{~kW}$ corresponding to $4.23 \%$ and $4.73 \%$ of the loss from PLIIHA. The search speed of the three applied methods is faster than PLIIHA because Npo and Niter are 10 and 30 for the three applied methods but 150
TABLE 1: Summary of results obtained by PSO, PPA, and TSA for the IEEE 15-bus distribution system with two capacitors.

\begin{tabular}{lccc}
\hline Method & PSO & PPA & TSA \\
\hline Best loss (kW) & 29.829 & 29.7361 & 29.6611 \\
Mean loss (kW) & 30.8437 & 32.4244 & 30.2492 \\
Worst loss (kW) & 31.9848 & 44.5112 & 32.6746 \\
SD & 0.5427 & 2.9974 & 0.7798 \\
\hline
\end{tabular}

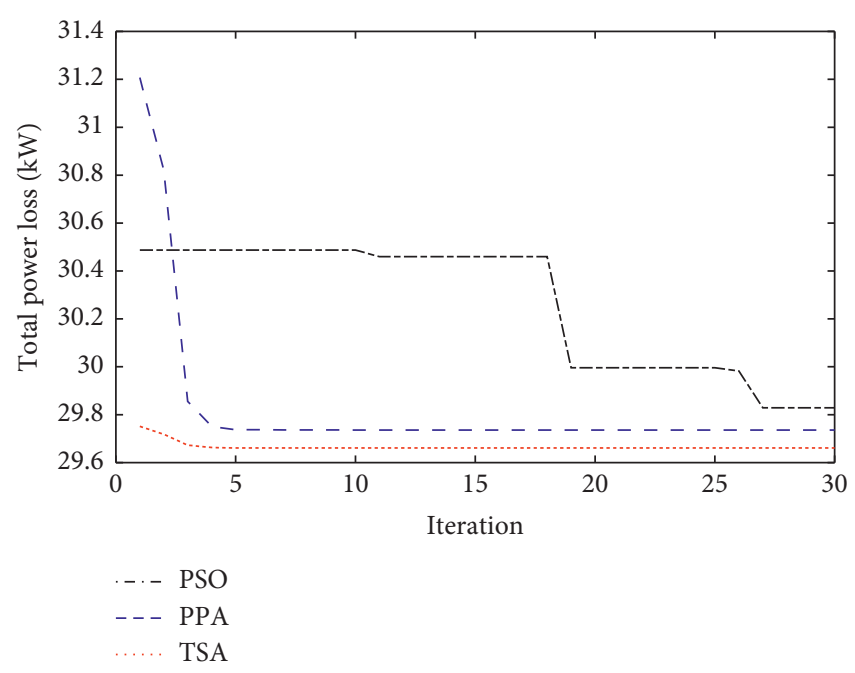

FIgURE 3: The best run of 50 runs obtained by PSO, PPA, and TSA for the IEEE 15-bus distribution system with two capacitors.

and 100 for PLIIHA. So, the three methods can be 45 times faster than PLIIHA in searching solution. The best total reactive power generation found by PLIIHA is $950 \mathrm{kVAr}$ but that found by PSO, PPA, and TSA is higher and equal to 1020 , 1008 , and $1109 \mathrm{kVAr}$. The values can see that PLIIHA was still searching far from the optimal solution with high quality.

5.2. Comparison and Discussion on Results Obtained for the IEEE 33-Bus Distribution System. In this section, a test system with 33 buses, called IEEE-33 bus distribution system [29], is employed to place capacitors. The compensation capacity at each load bus for increasing power factor to 0.9 is shown in Figure 9. The total capacity of all local capacitors is $615.0 \mathrm{kVAr}$. Three methods including PSO, PPA, and TSA are applied to place capacitors in the distribution lines. There are two study cases for the system in which two capacitors are placed in Case 1 and three capacitors are placed in Case 2. The comparison and discussion are as follows.

5.2.1. Case 1: Two Capacitors. In order to get fifty successful runs for PSO, PPA, and TSA, Npo and Niter are, respectively, set to 20 . The detail of the fifty runs is shown in Table 5. In addition, the best run and the loss of 50 runs are also plotted in Figures 10 and 11. The table can see the best loss of TSA is the lowest but its mean loss and worst loss are just the second best behind PPA. Figure 10 shows a much faster search potential of TSA than PSO and PPA since its loss at the 10th iteration is less than that of PSO and PPA at 


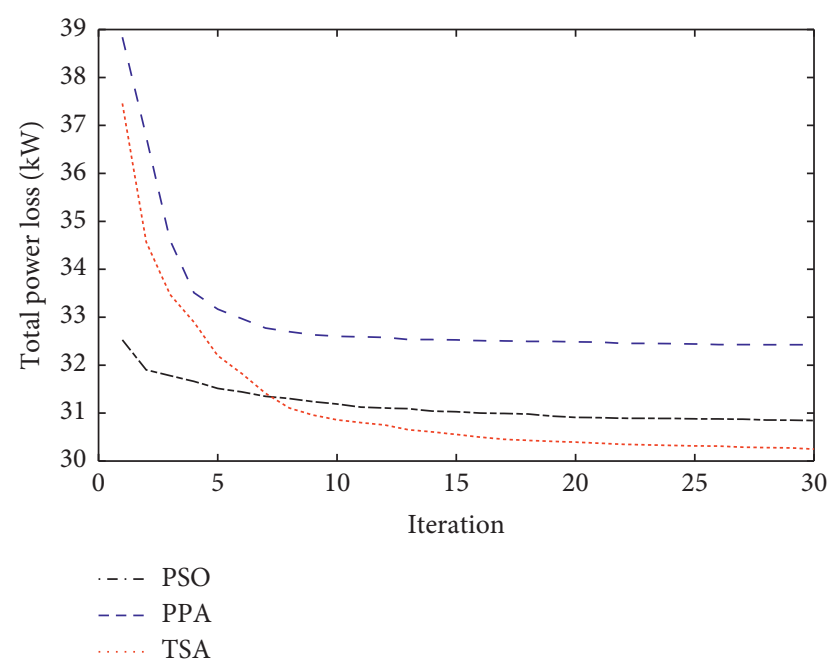

FIgURE 4: The mean run of 50 runs obtained by PSO, PPA, and TSA for the IEEE 15-bus distribution system with two capacitors.

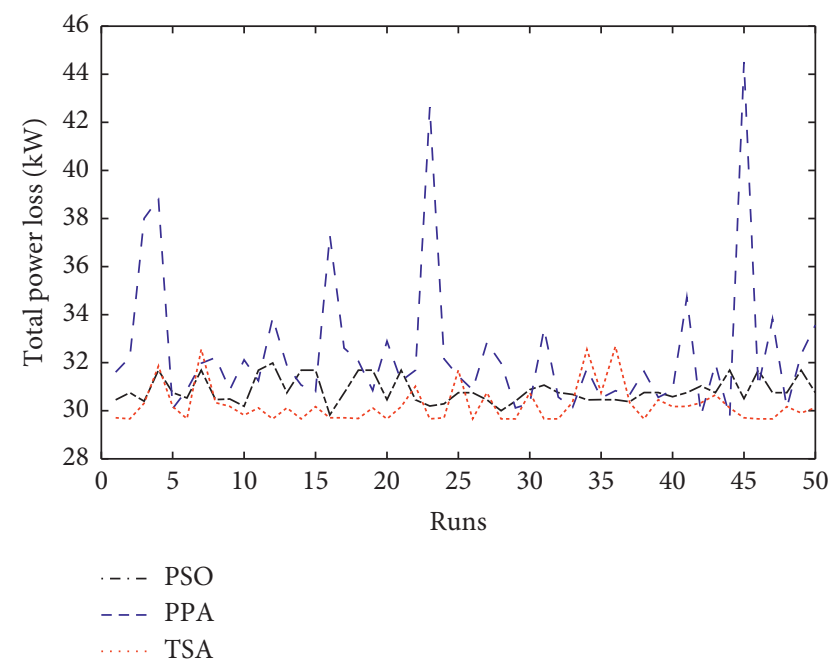

FIGURE 5: TAPL of 50 runs obtained by PSO, PPA, and TSA for the IEEE 15-bus distribution system with two capacitors.

the final iteration. Although the mean loss of TSA is not better than PPA, Figure 11 presents TSA can find fourteen solutions with smaller loss than the best one of PPA. The superiority of TSA over PSO is more significant. Thus, TSA is the best one among three applied methods for the case.

The comparisons with other methods for the case are shown in Table 6 . The loss with lowest value still reveals the best performance of TSA with less loss than PLA-MPSO [10] and NCAB [13] by 0.581 and $0.541 \mathrm{~kW}$, which are corresponding to $0.41 \%$ and $0.38 \%$ of the loss from the two compared methods. PSO and PPA also have the same improvement over the two compared methods because their loss is approximately as small as that of TSA. Furthermore, the most important issue is that the three applied methods are run by setting 20 to Npo and Niter but PLA-MPSO was run by assigning 10 and 200 to Npo and Niter. Clearly, the setting could support PLA-MPSO higher than 5 advantage times in finding the best site and capacity of the two capacitors. As a result, PLA-MPSO was still worse than TSA and PPA even worse than PSO. The analysis is evidence for the effectiveness of the reactive power compensation to increase power factor of all loads to 0.9 .

5.2.2. Case 2: Three Capacitors. In this case, three capacitors are placed in distribution lines of the system with 33 buses by running PSO, PPA, and TSA. Npo and Niter are, respectively, set to 20 and 50 for obtaining 50 successful runs. Table 7 and Figures 12 and 13 are built up for comparisons. The results are similar to those in Case 1 since TSA is still the best performance search tool with the smallest loss but its mean loss and maximum loss are not better than PPA. The best search run in Figure 12 shows a big challenge of finding the best solution of TSA as it cannot reach less loss than PPA for the first thirty-seven iterations. In spite of this disadvantage, TSA is also convergent to the best solution with less loss than the best one of PPA. The strong search of TSA can be confirmed by observing Figure 13 showing sixteen red points below the best blue point. This number can measure the outstanding search of TSA over PPA. All the values and figures can lead to the same evaluation of TSA and PSO that TSA is much more effective.

The robustness of TSA is competed to other ones by establishing Table 8. TSA can find less loss than three other methods including PLA-MPSO, NCAB, and NCAB by 0.41 , 0.69 , and $0.65 \mathrm{~kW}$. The loss reduction is equivalent to the improvement of performance with $0.3 \%, 0.5 \%$, and $0.47 \%$. PPA is also better than the three methods while PSO is worse than PLA-MPSO only. The exact computation indicates PSO reaches higher loss than PLA-MPSO by $0.03 \mathrm{~kW}$. However, it cannot state that PLA-MPSO wins PSO. Actually, PSO as well as PPA and TSA are run by setting $\mathrm{Npo}=20$ and Niter $=50$ but PLA-MPSO was executed by using Npo $=10$ and Niter $=200$. This comparison implies that TSA and PPA are much better than PLA-MPSO while PSO is able to find better solution than PLA-MPSO if we use the setting of PLAMPSO for PSO. Clearly, TSA is the best method and local compensation at each load is really effective for distribution systems.

5.3. Comparison and Discussion on Results Obtained for the IEEE 69-Bus Distribution System. In this section, the IEEE69 bus distribution system [29] is employed to place capacitors by using PSO, PPA, and TSA. The compensation capacity at each load bus is presented in Figure 14. The total capacity of all local capacitors is $853.45 \mathrm{kVAr}$. Three methods including PSO, PPA, and TSA are applied to place capacitors in the distribution lines. Similar to the two systems above, Case 1 with the placement of two capacitors and Case 2 with the placement of three capacitors in distribution lines are executed and discussed in the following sections.

5.3.1. Case 1: Two Capacitors. For reaching the summarized results from 50 successful runs shown in Table 9, the three applied methods are run by setting $\mathrm{Npo}=10$ and Niter $=30$. 
TABLe 2: Result comparison for the IEEE 15-bus distribution system with two capacitors.

\begin{tabular}{|c|c|c|c|c|c|c|}
\hline Method & Capacitor site & Size (kVAr) & Total kVAr & Total loss $(\mathrm{kW})$ & Npo & Niter \\
\hline Two-step method [1] & 3,6 & 805,388 & 1193 & 32.6 & - & - \\
\hline WCPSO [5] & 3,6 & 871,321 & 1192 & 32.7 & - & - \\
\hline LSFACA [9] & 4,6 & 630,410 & 1040 & 36.81 & - & - \\
\hline PSO & 3,7 & 383,144 & $527+527=1054$ & 29.828 & 10 & 30 \\
\hline PPA & 4,6 & 295,202 & $497+527=1024$ & 29.736 & 10 & 30 \\
\hline TSA & 4,6 & 340,213 & $553+527=1080$ & 29.661 & 10 & 30 \\
\hline
\end{tabular}

TABLE 3: Summary of results obtained by PSO, PPA, and TSA for the IEEE 15-bus distribution system with three capacitors.

\begin{tabular}{lccc}
\hline Method & PSO & PPA & TSA \\
\hline Best loss (kW) & 29.809 & 29.654 & 29.202 \\
Mean loss (kW) & 31.220 & 33.602 & 30.203 \\
Worst loss (kW) & 36.437 & 62.432 & 32.084 \\
SD & 1.477 & 6.057 & 0.686 \\
\hline
\end{tabular}

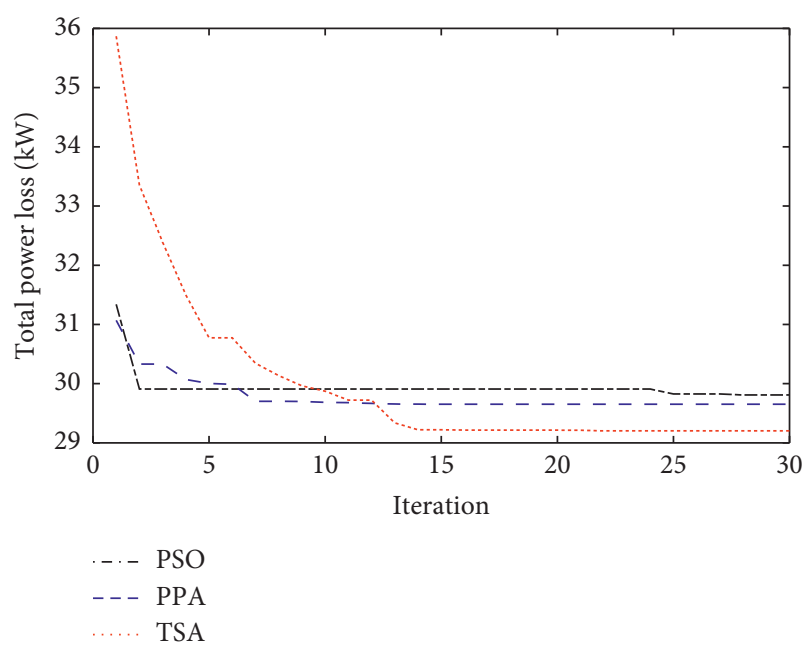

Figure 6: The best run of 50 runs obtained by PSO, PPA, and TSA for the IEEE 15-bus distribution system with three capacitors.

The minimum loss of TSA is the best value but its mean and maximum losses are not the best but those of PSO are the best. Figure 15 shows the fastest solution seeking procedure of TSA once its solution found at the seventeenth iteration has less loss than that from PSO and TSA at the end of the search procedure. Figure 16 presents TSA can get eight better solutions and over ten better solutions than the best one of PSO and PPA, respectively. Thus, TSA is still the best one for Case 1 of the system.

The comparisons of PSO, PPA, and TSA with two other methods in $[10,13]$ are shown in Table 10. The three applied methods can reach better loss than PLA-MPSO and NCAB. The loss reduction that TSA, PSO, and PPA can get as compared to the two methods is $1.59 \mathrm{~kW}, 1.514 \mathrm{~kW}$, and $1.45 \mathrm{~kW}$. The saving loss is equal to the improvement of $1.1 \%, 1.03 \%$, and $0.99 \%$. PLA-MPSO was run by adopting $\mathrm{Npo}=10$ and Niter $=200$, which are about 7 times slower than TSA, PSO, and PPA. Clearly, TSA is much more effective than the method. However, it should be emphasized

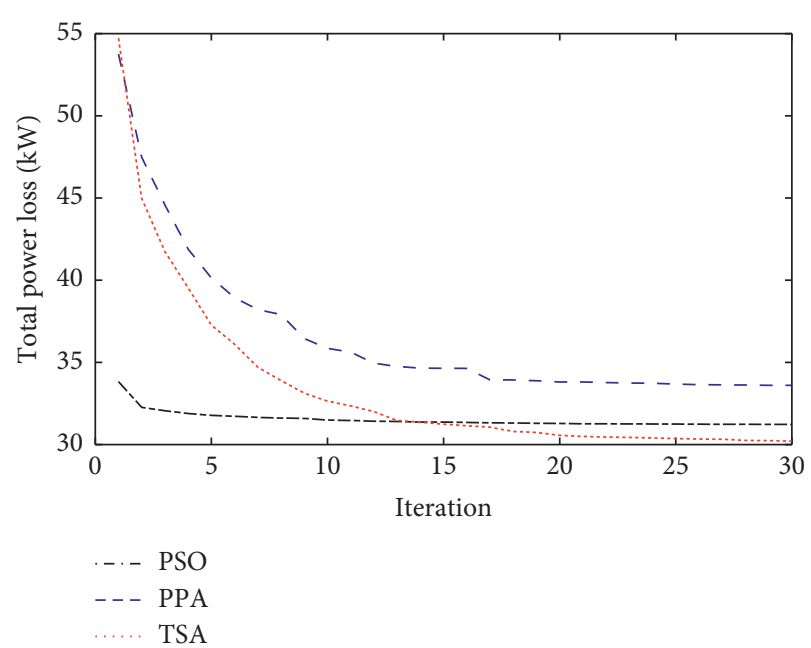

FIGURE 7: The mean run of 50 runs obtained by PSO, PPA, and TSA for the IEEE 15-bus distribution system with three capacitors.

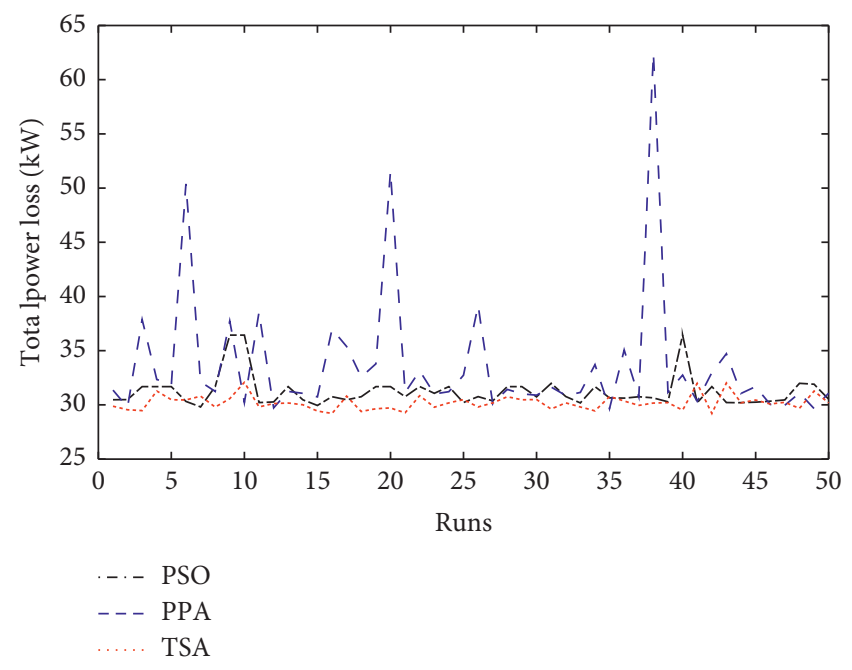

FIgURE 8: TAPL of 50 runs obtained by PSO, PPA, and TSA for the IEEE 15-bus distribution system with three capacitors.

that this method used 200 iterations while PSO used 30 iterations but its result is still worse than that of PSO. Furthermore, PLA-MPSO was the combination of power loss analysis method and the modified version of PSO (MPSO) in which MPSO was more effective than PSO. The analysis reflects the high effectiveness of combination of local compensation at each load to increase power factor and the compensation in distribution lines. 
TABLE 4: Result comparison for the IEEE 15-bus distribution system with three capacitors.

\begin{tabular}{|c|c|c|c|c|c|c|}
\hline Method & Capacitor site & Size (kVAr) & Total kVAr & Total loss $(\mathrm{kW})$ & Npo & Niter \\
\hline PLIIHA [8] & $6,11,15$ & $350,300,300$ & 950 & 31.1255 & 150 & 100 \\
\hline PSO & $4,6,7$ & $0,321,172$ & $493+527=1020$ & 29.809 & 10 & 30 \\
\hline PPA & $4,6,12$ & $136,238,107$ & $481+527=1008$ & 29.654 & 10 & 30 \\
\hline TSA & $4,6,11$ & $238,201,143$ & $582+527=1109$ & 29.202 & 10 & 30 \\
\hline
\end{tabular}

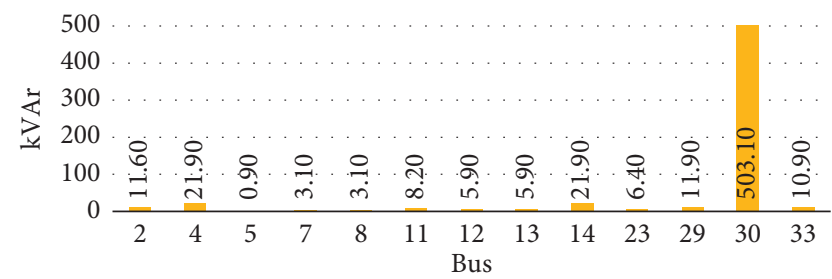

FIgure 9: Compensation capacity at loads in the IEEE 33-bus distribution system.

TABLE 5: Summary of results obtained by PSO, PPA, and TSA for the IEEE 33-bus distribution system with two capacitors.

\begin{tabular}{lccc}
\hline Method & PSO & PPA & TSA \\
\hline Best loss (kW) & 141.3707 & 141.3795 & 141.3591 \\
Mean loss (kW) & 142.9242 & 142.1692 & 142.4312 \\
Worst loss (kW) & 146.0363 & 144.0607 & 145.1886 \\
SD & 1.2347 & 0.6575 & 0.67 \\
\hline
\end{tabular}

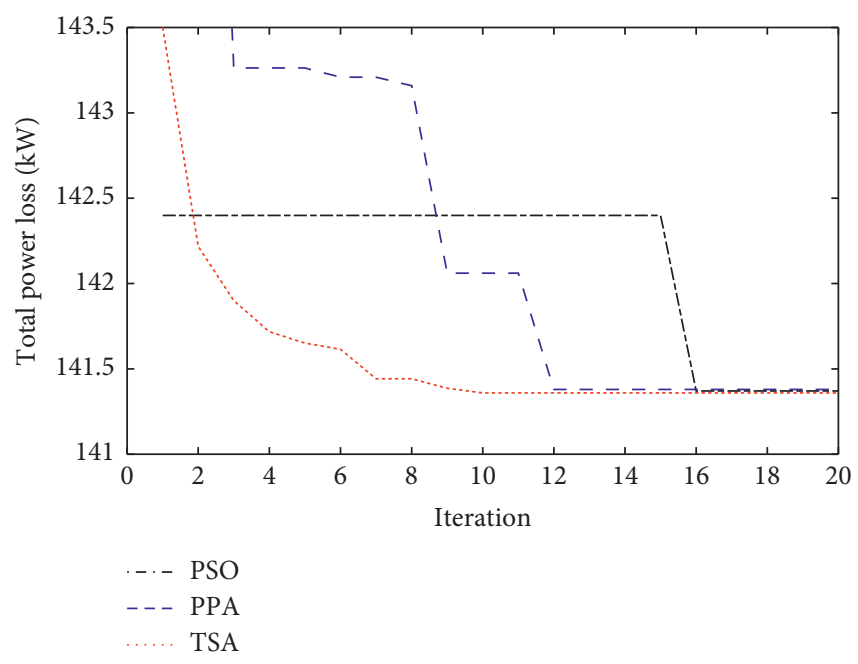

FIgURE 10: The best run of 50 runs obtained by PSO, PPA, and TSA for the IEEE 33-bus distribution system with two capacitors.

5.3.2. Case 2: Three Capacitors. In Case 2, three capacitors are placed in distribution lines by running PSO, PPA, and TSA with the setting of $\mathrm{Npo}=30$ and Niter $=60$. The obtained results are summarized in Table 11. TSA is still the best method with the lowest minimum loss. The best run in Figure 17 shows the good search of TSA, which is much faster than that of PSO and PPA. TSA can find a better solution than PSO and PPA and even the solution of TSA at fifteenth iteration is better than the final solution of PSO and PPA. Observing Figure 18 shows that TSA can find three better solutions than the best one of PPA and over six better

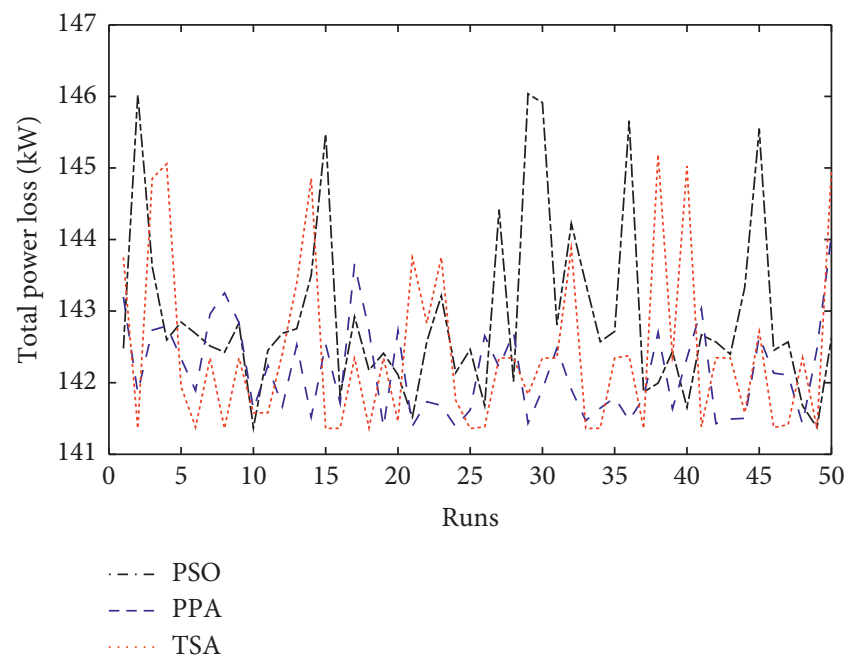

FIgURE 11: TAPL of 50 runs obtained by PSO, PPA, and TSA for the IEEE 33-bus distribution system with two capacitors.

solutions than the best one of PSO. For the case of the 69-bus system, TSA cannot reach the same good results as for other systems above with 15 buses and 33 buses.

The comparisons with other methods for the case are shown in Table 12. The loss comparison indicates that TSA can find the location and size of capacitors with less loss than other methods from $0.96 \mathrm{~kW}$ to $12.38 \mathrm{~kW}$, which are corresponding to the improvement from $0.66 \%$ to $7.9 \%$. Both PSO and PPA also reach better loss than these compared methods. The two methods can get the loss reduction from $0.68 \mathrm{~kW}$ and $0.95 \mathrm{~kW}$ to $12.1 \mathrm{KW}$ and $12.37 \mathrm{~kW}$. The loss reduction is equivalent to the improvement from $0.47 \%$ and $0.66 \%$ to $7.7 \%$ and $7.9 \%$. Clearly, the improvement is significant. The method with the highest power loss of $156.62 \mathrm{~kW}$ is GA, which was run by adopting Npo $=60$ and Niter $=100$, while the method with the second best loss (behind TSA, PSO, and PPA) of $145.2 \mathrm{~kW}$ is PLA-MPSO, which was successfully applied by setting $\mathrm{Npo}=10$ and Niter $=200$. In addition to the worst method and the second best method, other ones were also run with high values for Npo and Niter. In fact, Npo and Niter are, respectively, 2500 and 750 for GSA, 50 and 100 for TLBA and MSA, and 20 and 200 for FPA. About the settings, the three applied methods are the most powerful tools with the lowest values for control parameter, Npo $=30$ and Niter $=60$. Hence, it leads to a conclusion that TSA is the best performance method with the smallest loss and the fastest speed and the combination of the local compensation at each load to increase power factor to 0.9 and the compensation in distribution lines is really useful for reaching loss reduction. 
TABLE 6: Result comparison for the IEEE 33-bus distribution system with two capacitors.

\begin{tabular}{|c|c|c|c|c|c|c|}
\hline Method & Capacitor site & Size (kVAr) & Total kVAr & Total loss $(\mathrm{kW})$ & Npo & Niter \\
\hline PLA-MPSO [10] & 12,30 & 403,1040 & 1470 & 141.94 & 10 & 200 \\
\hline NCAB [13] & 13,30 & 405,1052 & 1457 & 141.9 & NR & NR \\
\hline PSO & 12,30 & 431,531 & $962+615=1577$ & 141.371 & 20 & 20 \\
\hline PPA & 10,30 & 442,523 & $965+615=1580$ & 141.379 & 20 & 20 \\
\hline TSA & 11,30 & 434,527 & $961+615=1576$ & 141.359 & 20 & 20 \\
\hline
\end{tabular}

TABLE 7: Summary of results obtained by PSO, PPA, and TSA for the IEEE 33-bus distribution system with three capacitors.

\begin{tabular}{lccc}
\hline Method & PSO & PPA & TSA \\
\hline Best loss (kW) & 138.4001 & 138.1749 & 137.9647 \\
Mean loss (kW) & 141.0291 & 139.861 & 139.9487 \\
Worst loss (kW) & 146.6033 & 143.1531 & 146.4281 \\
SD & 1.4883 & 1.25 & 1.387 \\
\hline
\end{tabular}

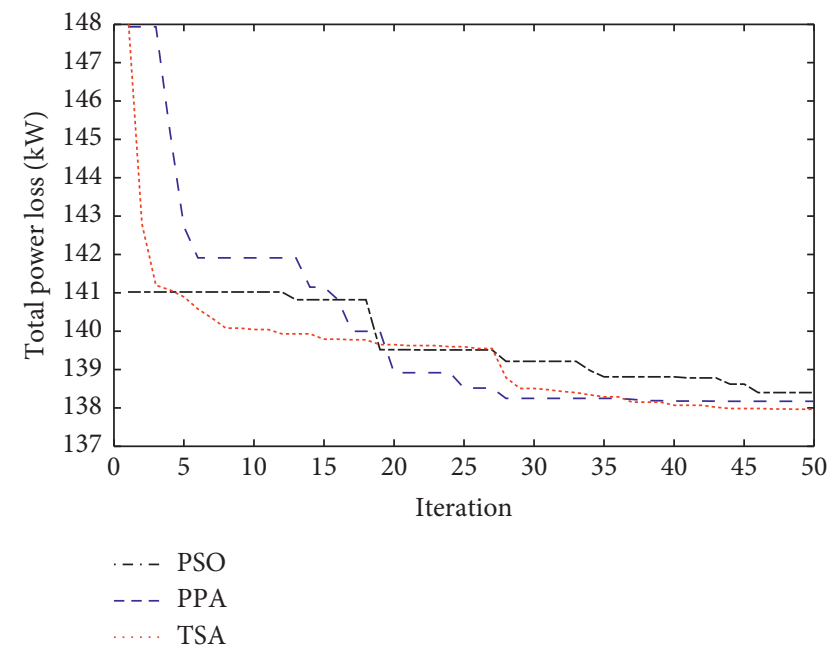

FIGURE 12: The best run of 50 runs obtained by PSO, PPA, and TSA for the IEEE 33-bus distribution system with three capacitors.

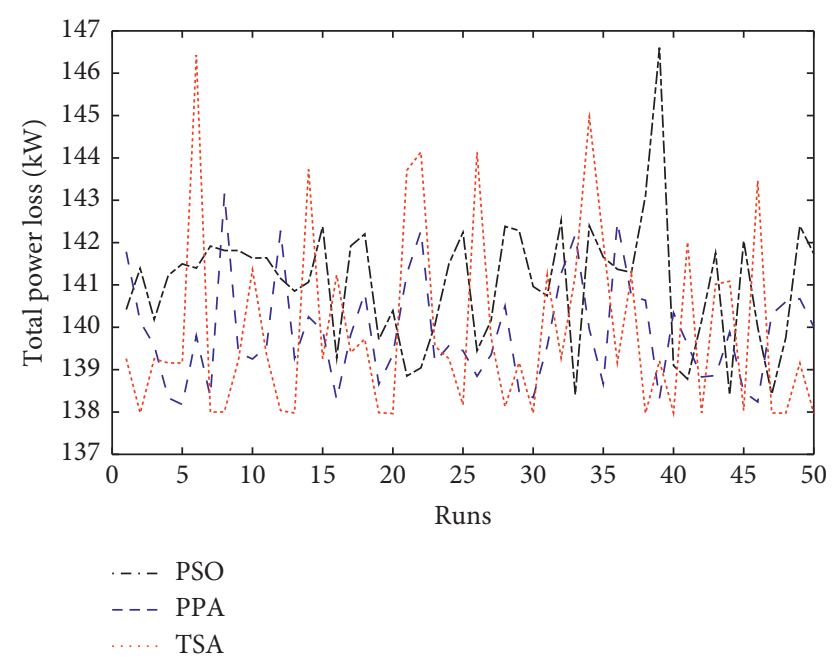

Figure 13: TAPL of 50 runs obtained by PSO, PPA, and TSA for the IEEE 33-bus distribution system with three capacitors.
5.4. Comparison and Discussion on Results Obtained for the IEEE 85-Bus Distribution System. In this section, the largest system with 85 buses is employed to place capacitors for loss reduction. Data of the system are reported in [32]. To increase power factor of all loads to 0.9 , total capacity is 1377.23 kVAr. The compensation capacity at each load bus is presented in Figure 19. Two study cases of the system are the placement of three capacitors in Case 1 and the placement of four capacitors in Case 2. The results and discussion of the cases are as follows.

5.4.1. Case 1: Three Capacitors. In order to run PSO, PPA, and TSA, Npo and Niter are set to 30 and 60, respectively. The summary of results is shown in Table 13 while the best run and the loss of 50 runs are plotted in Figures 20 and 21. The result comparisons can give the same evaluation as previous sections; that is, TSA can reach the lowest loss but the mean loss and the worst loss are not the best values among three applied methods. The minimum loss of TSA is $143.1539 \mathrm{~kW}$ while that of PSO and PPA is, respectively, $144.0476 \mathrm{~kW}$ and $143.2711 \mathrm{~kW}$. For measuring the search speed of methods, Figure 20 shows the solution found by TSA at the 35th iteration has smaller loss than that of PSO and TSA at the final iteration. Figure 21 shows that TSA can find seven and over thirty better solutions than the best one of PPA and PSO, respectively. Thus, TSA continues to be the best one among three applied methods for the case.

The performance of TSA and the proposed compensation method can be evaluated by using Table 14 . The loss still shows the best performance of TSA once it can reach loss reduction to $9.097 \mathrm{~kW}$ and $8.654 \mathrm{~kW}$ as compared to BFA and FPA. The reduction of loss is corresponding to $5.98 \%$ and $5.7 \%$, respectively. However, TSA is the fastest method with the setting of $\mathrm{Npo}=30$ and Niter $=60$ but the setting is $\mathrm{Npo}=100$ and Niter $=50$ for BFA, and $\mathrm{Npo}=20$ and Niter $=200$ for FPA. The settings indicate that TSA can be about three times and two times faster than BFA and FPA, respectively. PSO and PPA are also more effective than BFA and FPA with the loss reduction of $8.202 \mathrm{~kW}$ and $7.759 \mathrm{KW}$ (for comparison with PSO) and $8.98 \mathrm{~kW}$ and $8.537 \mathrm{~kW}$ (for comparison with PPA). The loss reduction indicates that the improvement of PSO and PPA over the two methods is $5.39 \%$ and $5.11 \%$, and $5.9 \%$ and $5.62 \%$, respectively. So, it can conclude that TSA is much better than other previous methods thanks to its strong search ability and the proposed compensation method. 
TABLE 8: Result comparison for the IEEE 33-bus distribution system with three capacitors.

\begin{tabular}{|c|c|c|c|c|c|c|}
\hline Method & Capacitor site & Size (kVAr) & Total kVAr & Total loss $(\mathrm{kW})$ & Npo & Niter \\
\hline PLA-MPSO [10] & $13,24,30$ & $360,510,1020$ & 1890 & 138.37 & 10 & 200 \\
\hline NCAB [13] & $13,25,30$ & $383,386,1000$ & 1769 & 138.65 & NR & NR \\
\hline NCAB [14] & $12,24,30$ & $500,500,1000$ & 2000 & 138.61 & NR & NR \\
\hline PSO & $12,25,31$ & $417,463,434$ & $1314+615=1929$ & 138.40 & 20 & 50 \\
\hline PPA & $13,24,30$ & $319,487,582$ & $1388+615=2003$ & 138.17 & 20 & 50 \\
\hline TSA & $12,24,30$ & $393,530,491$ & $1414+615=2029$ & 137.96 & 20 & 50 \\
\hline
\end{tabular}
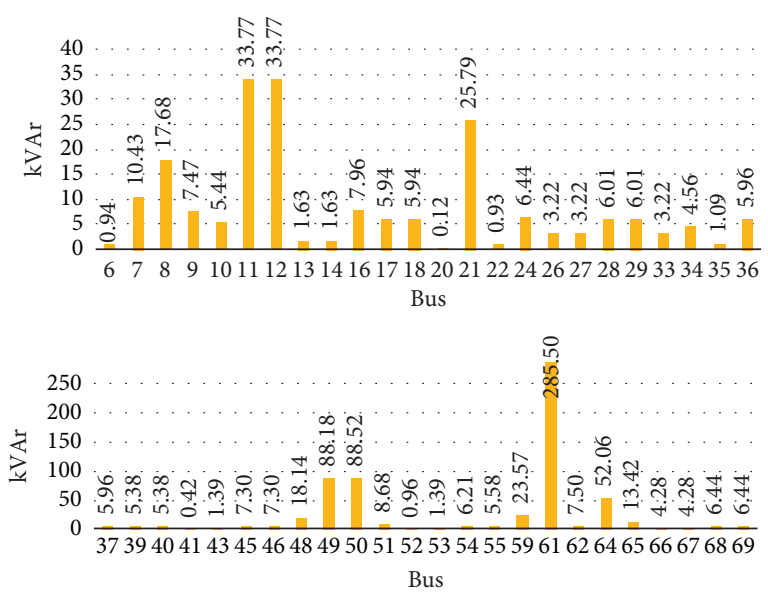

FIGURE 14: Compensation capacity at loads in the IEEE 69-bus distribution system.

TABLE 9: Summary of results obtained by PSO, PPA, and TSA for the IEEE 69-bus distribution system with two capacitors.

\begin{tabular}{lccc}
\hline Method & PSO & PPA & TSA \\
\hline Best loss (kW) & 144.986 & 145.053 & 144.901 \\
Mean loss (kW) & 147.354 & 149.336 & 147.444 \\
Worst loss (kW) & 149.234 & 161.118 & 169.790 \\
SD & 0.978 & 3.809 & 3.777 \\
\hline
\end{tabular}

5.4.2. Case 2: Four Capacitors. In this section, four capacitors are placed in the IEEE 85-bus system by running PSO, PPA, and TSA with the settings of $\mathrm{Npo}=30$ and Niter $=100$. The results are summarized in Table 15 and Figures 22 and 23. The values shown in Table 15 and Figure 22 indicate the best loss of TSA and the best search ability among the three methods. Figure 23 shows the better stability of TSA and PPA over PSO but TSA can reach many solutions with less loss than that of PPA. So, TSA is the best method among the three applied methods.

The comparison with mixed integer nonlinear programming (MINP) [24] for the study case is reported in Table 16. TSA, PSO, and PPA can reach less loss than MINP by 17.198 , 16.336 , and $17.117 \mathrm{~kW}$, which are equal to $10.76 \%, 10.21 \%$, and $10.7 \%$ of total loss from MINP. The values are really significant for a large-scale system as the IEEE 85-bus distribution system. So, the proposed compensation method and the applied TSA

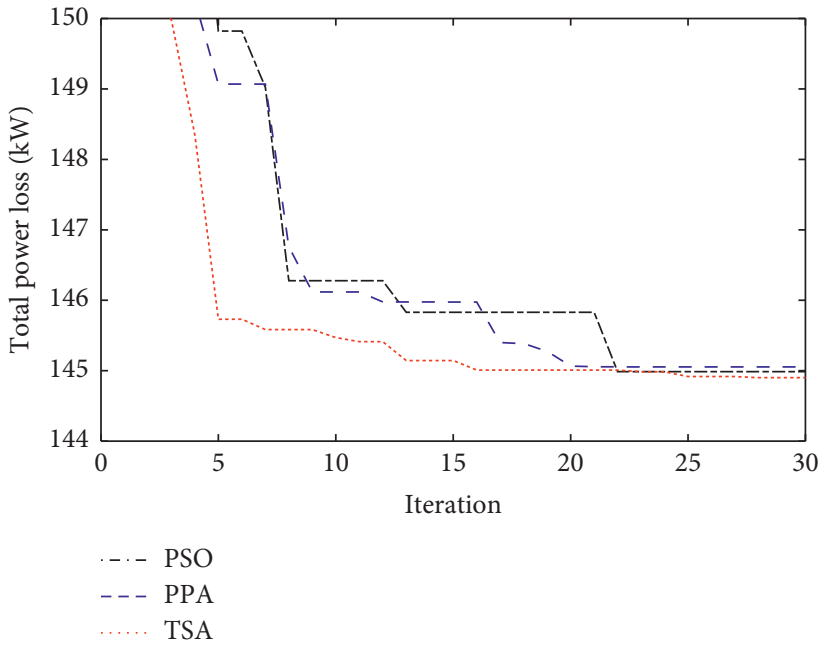

FIGURE 15: The best run of 50 runs obtained by PSO, PPA, and TSA for the IEEE 69-bus distribution system with two capacitors.

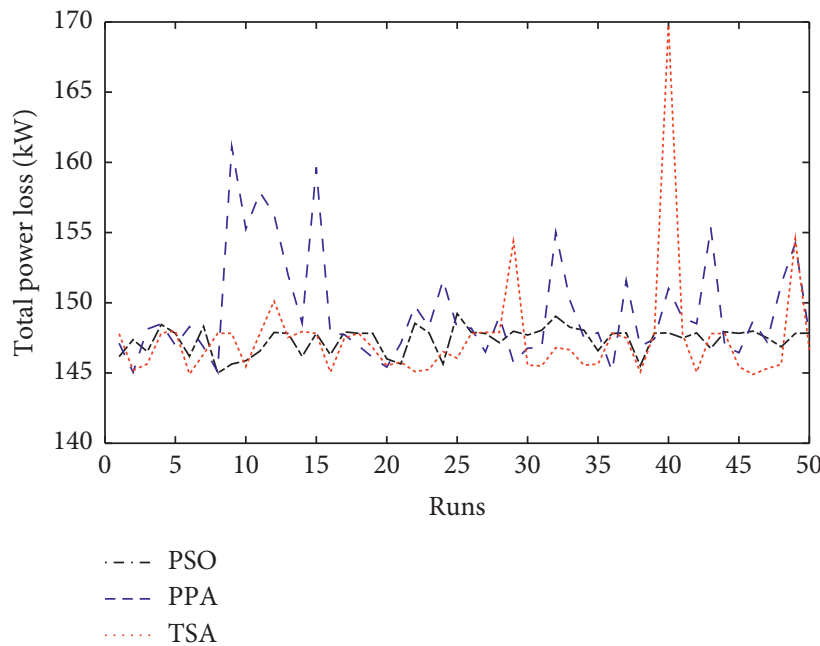

FIgURE 16: TAPL of 50 runs obtained by PSO, PPA, and TSA for the IEEE 69-bus distribution system with two capacitors.

method are really effective for the loss reduction. Although MINP is not a metaheuristic algorithm, the search speed of this one can be ensured not to be as short as the three applied methods because, as shown in [24], the method was based on configuration analysis of distribution systems. 
TABLE 10: Result comparison for the IEEE 69-bus distribution system with two capacitors.

\begin{tabular}{|c|c|c|c|c|c|c|}
\hline Method & Capacitor site & Size (kVAr) & Total kVAr & Total loss $(\mathrm{kW})$ & Npo & Niter \\
\hline PLA-MPSO [10] & 18,61 & 350,1240 & 1590 & 146.5 & 10 & 200 \\
\hline NCAB [13] & 17,61 & 356,1224 & 1580 & 146.5 & NR & NR \\
\hline PSO & 16,61 & 313,870 & $1183+853=2036$ & 144.986 & 10 & 30 \\
\hline PPA & 17,61 & 252,943 & $1195+853=2048$ & 145.053 & 10 & 30 \\
\hline TSA & 17,61 & 272,883 & $1155+853=2008$ & 144.901 & 10 & 30 \\
\hline
\end{tabular}

TABLE 11: Summary of results obtained by PSO, PPA, and TSA for the IEEE 69-bus distribution system with three capacitors.

\begin{tabular}{lccc}
\hline Method & PSO & PPA & TSA \\
\hline Best loss $(\mathrm{kW})$ & 144.521 & 144.247 & 144.238 \\
Mean loss $(\mathrm{kW})$ & 145.811 & 144.946 & 145.692 \\
Worst loss $(\mathrm{kW})$ & 147.819 & 147.784 & 147.861 \\
SD & 0.890 & 0.624 & 1.006 \\
\hline
\end{tabular}

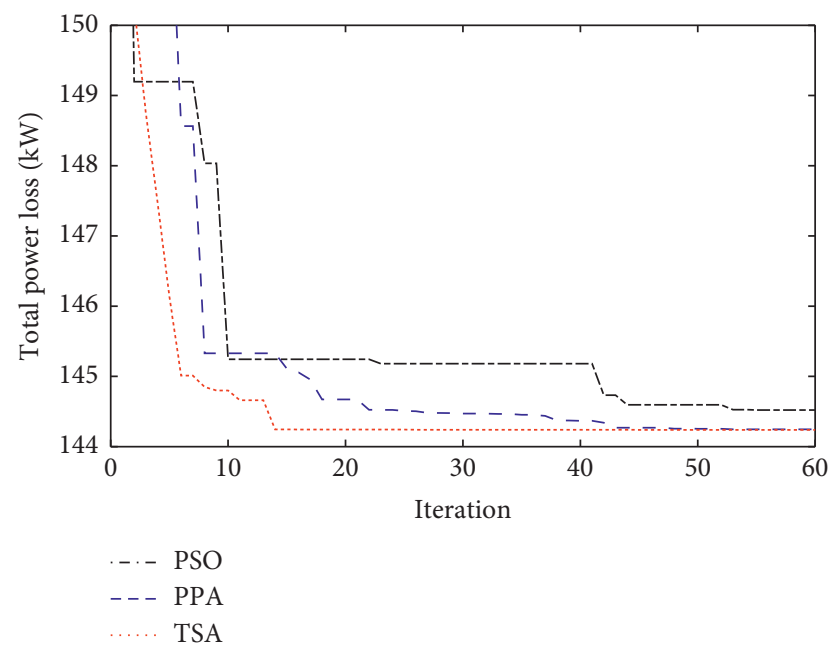

FIGURE 17: The best run of 50 runs obtained by PSO, PPA, and TSA for the IEEE 69-bus distribution system with three capacitors.

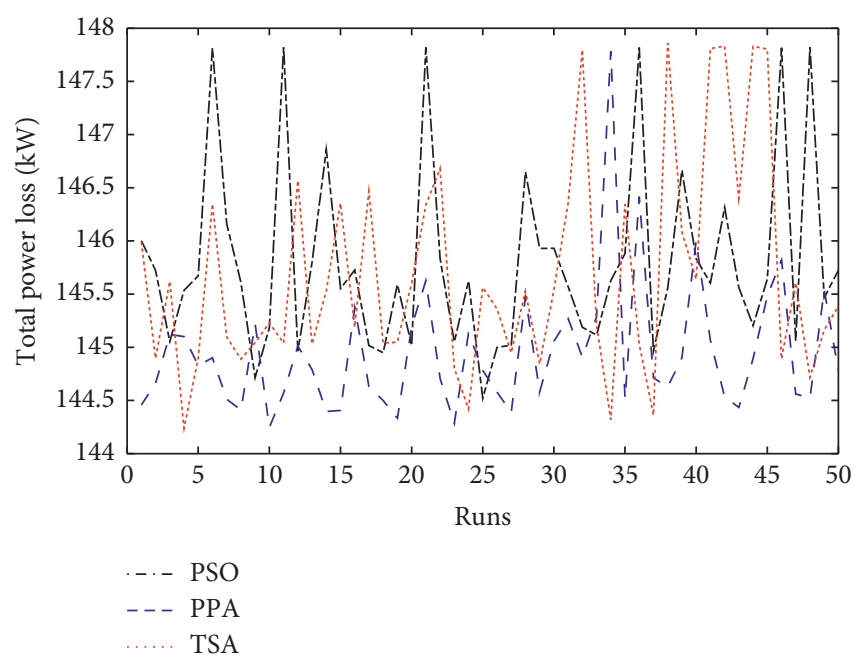

FIgURE 18: TAPL of 50 runs obtained by PSO, PPA, and TSA for the IEEE 69-bus distribution system with three capacitors.
5.5. Discussion on the Improvement Level of TSA over PSO and $P P A$. In this section, we investigate of the improvement level of TSA over PSO and PPA as dealing with the reactive power compensation in the four distribution systems. A statistical test, called Wilcoxon rank [40], is employed to evaluate the whole fifty valid solutions found by TSA, PPA, and PSO. The test is run for each two methods including TSA and PPA, and TSA and PSO, and obtained results indicate the considerable improvement level of TSA. To run Wilcoxon test, the significant level is set to 0.05 and the fifty loss values of TSA and PPA, and TSA and PSO are added to determine $Z, W$, and $p$ parameters. As pointed in $[41,42]$, values of $p$ parameter are taken to decide the considerable improvement level and 0.05 is the boundary to separate the performance of TSA into significant or insignificant improvement level. If $p$ is smaller than 0.05 , the improvement of TSA over another compared method is significant and vice versa. After running Wilcoxon test for TSA, PSO, and PPA, the results including $Z, W$, and $p$ are presented in Table 17. In addition, the improvement level is also evaluated and added in the last column based on the values of $p$. For the first system with 15 buses, $p$ is less than 0.00001 for all cases and the improvement level of TSA over PSO as well as PPA is significant for Case 1 and Case 2. Unlikely, TSA cannot reach the same considerable improvement level for remaining systems with 33, 69, and 85 buses. For system with 33 buses, $p<0.0232$ and $p=0.01552$ for the statistical test with PSO indicate that the improvement level of TSA over PSO is considerable but $p=0.267$ and 0.86502 for the statistical test with PPA indicate that the improvement level of TSA over PPA is insignificant. The improvement of TSA over PSO is insignificant for the system with 69 buses but significant for the system with 85 buses. Unlikely, the improvement of TSA over PPA is significant for the system with 69 buses but insignificant for the system with 85 buses.

The statistical test clarifies the improvement level of the major method TSA over PSO and PPA when implementing the three methods for fifty successful runs. Although TSA cannot reach the considerable improvement level for all study cases, it always reaches the major target of minimizing power loss among the three applied methods and among a number of compared methods. So, it can be stated that the combination of reactive power compensation at loads and at node in distribution lines can reach the high achievements thanks to the application of TSA. 
TABLE 12: Result comparison for the IEEE 69-bus distribution system with three capacitors.

\begin{tabular}{|c|c|c|c|c|c|c|}
\hline Method & Capacitor site & Size (kVAr) & Total kVAr & Total loss $(\mathrm{kW})$ & Npo & Niter \\
\hline Two-step method [4] & $19,62,63$ & $225,900,250$ & 1350 & 148.91 & NR & NR \\
\hline WCPSO [5] & $46,47,50$ & $241,365,1015$ & 1621 & 152.48 & - & - \\
\hline PLA-MPSO [10] & $11,18,61$ & $330,250,1190$ & 1770 & 145.2 & 10 & 200 \\
\hline NCAB [13] & $12,21,61$ & $320,226,210$ & 1756 & 145.3 & NR & NR \\
\hline NCAB [15] & $11,21,61$ & $368,231,1196$ & 1795 & 145.21 & NR & NR \\
\hline GA [20] & $59,61,64$ & $100,700,800$ & 1600 & 156.62 & 60 & 100 \\
\hline GSA [27] & $13,15,26$ & $150,1050,150$ & 1350 & 145.9 & 2500 & 750 \\
\hline TLBA [28] & $12,61,64$ & $600,1050,150$ & 1800 & 146.35 & 50 & 100 \\
\hline FPA [31] & $11,22,64$ & $450,150,1350$ & 1950 & 145.86 & 20 & 200 \\
\hline CSA [33] & $18,61,65$ & $350,1150,65$ & 1565 & 146.1 & 25 & - \\
\hline MSA [34] & $12,21,61$ & $450,150,1200$ & 1800 & 145.41 & 50 & 100 \\
\hline PSO & $16,61,69$ & $199,856,186$ & $1241+853=2094$ & 144.521 & 30 & 60 \\
\hline PPA & $11,22,61$ & $305,169,849$ & $1323+853=2176$ & 144.247 & 30 & 60 \\
\hline TSA & $11,17,61$ & $281,186,847$ & $1314+853=2167$ & 144.238 & 30 & 60 \\
\hline
\end{tabular}
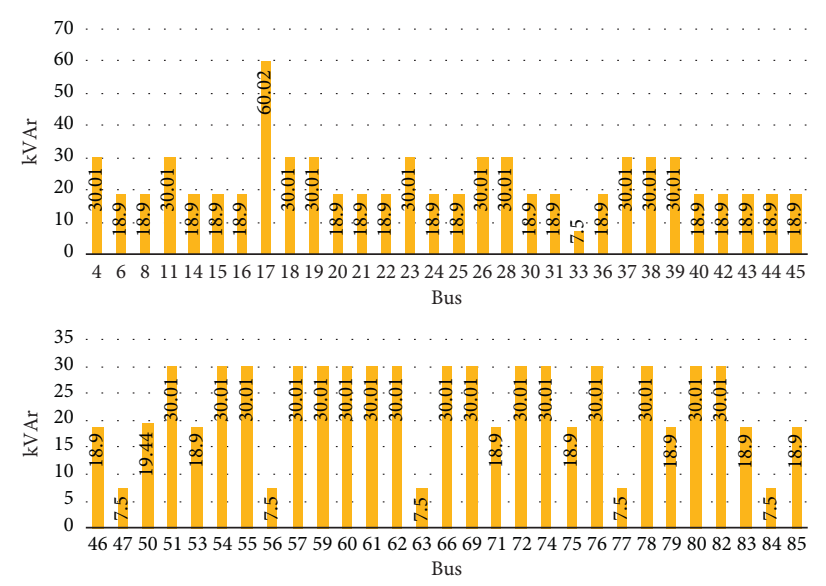

FIGURE 19: Compensation capacity at loads in the IEEE 85-bus distribution system.

TABLE 13: Summary of results obtained by PSO, PPA, and TSA for the IEEE 85-bus distribution system with three capacitors.

\begin{tabular}{lccc}
\hline Method & PSO & PPA & TSA \\
\hline Best loss $(\mathrm{kW})$ & 144.0476 & 143.2711 & 143.1539 \\
Mean loss (kW) & 146.3503 & 144.1091 & 144.4088 \\
Worst loss (kW) & 149.9005 & 145.5883 & 149.5757 \\
SD & 1.368 & 0.5624 & 1.1508 \\
\hline
\end{tabular}

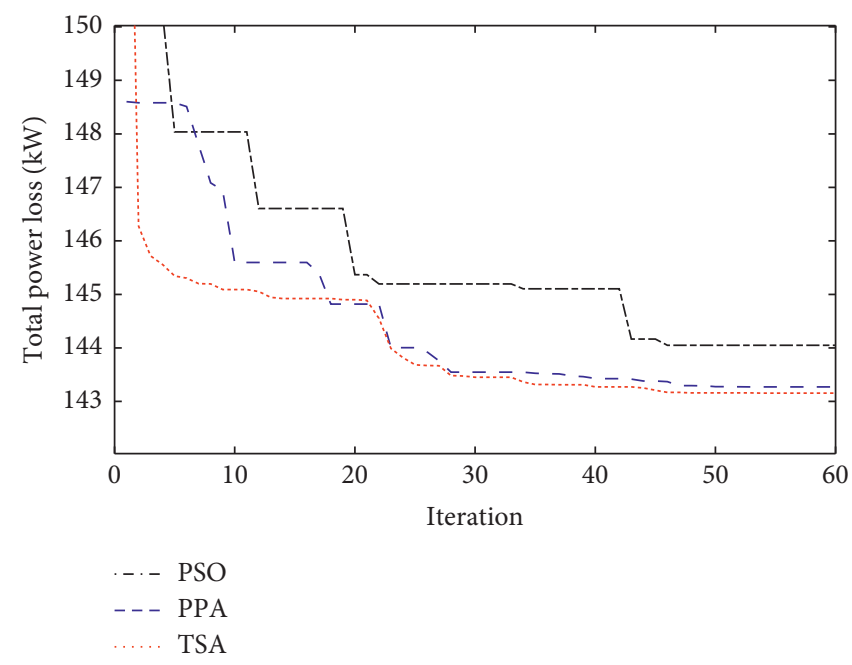

FIGURE 20: The best run of 50 runs obtained by PSO, PPA, and TSA for the IEEE 85-bus distribution system with three capacitors. 


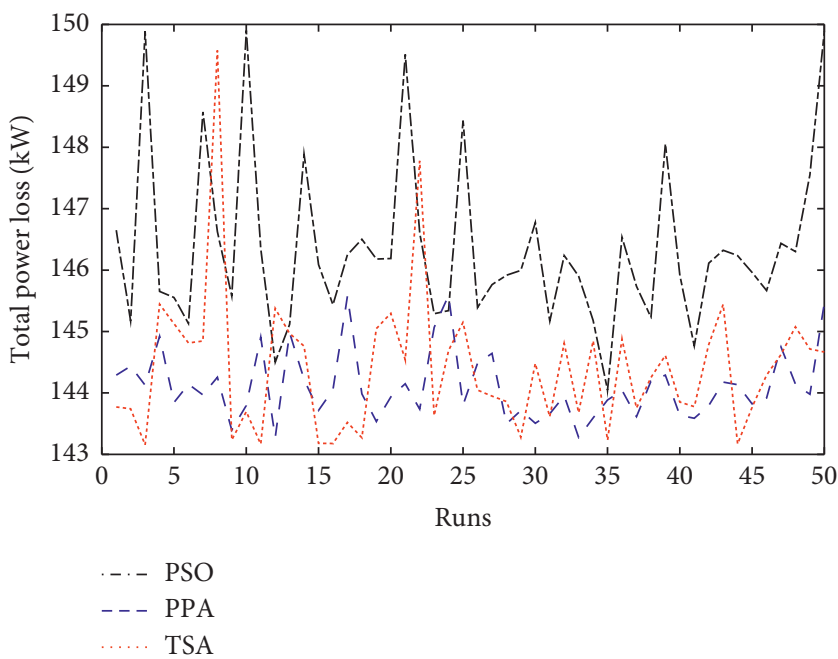

FIgURE 21: TAPL of 50 runs obtained by PSO, PPA, and TSA for the IEEE 85-bus distribution system with three capacitors.

TABLE 14: Result comparison for the IEEE 85-bus distribution system with three capacitors.

\begin{tabular}{|c|c|c|c|c|c|c|}
\hline Method & Capacitor site & Size (kVAr) & Total kVAr & Total loss $(\mathrm{kW})$ & Npo & Niter \\
\hline BFA [26] & $9,34,60$ & $840,660,650$ & 2150 & 152.25 & 100 & 50 \\
\hline FPA [31] & $8,36,72$ & $1200,600,600$ & 2400 & 151.807 & 20 & 200 \\
\hline PSO & $9,52,69$ & $657,259,231$ & $1147+1377=2524$ & 144.048 & 30 & 60 \\
\hline PPA & $8,34,68$ & $663,317,182$ & $1162+1377=2539$ & 143.27 & 30 & 60 \\
\hline TSA & $9,34,67$ & $562,324,248$ & $1134+1377=2511$ & 143.153 & 30 & 60 \\
\hline
\end{tabular}

TABLE 15: Summary of results obtained by PSO, PPA, and TSA for the IEEE 85-bus distribution system with four capacitors.

\begin{tabular}{lccc}
\hline Method & PSO & PPA & TSA \\
\hline Min. TAPL $(\mathrm{kW})$ & 143.5341 & 142.7538 & 142.6718 \\
Mean TAPL $(\mathrm{kW})$ & 145.9785 & 143.4734 & 143.8302 \\
Max. TAPL $(\mathrm{kW})$ & 152.2482 & 145.0783 & 147.0468 \\
Std. dev. & 1.5441 & 0.4953 & 0.9011 \\
\hline
\end{tabular}

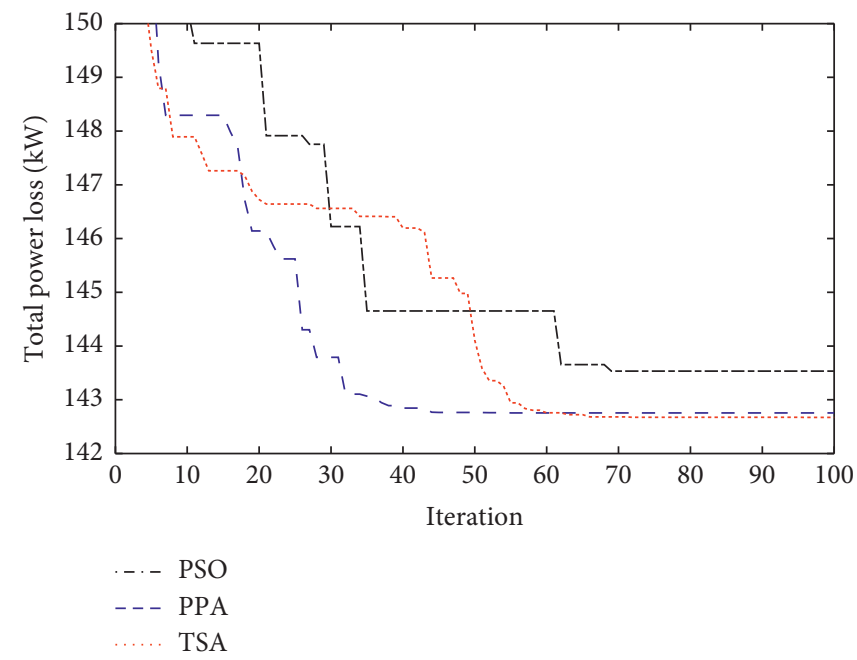

FIGURE 22: The best run of 50 runs obtained by PSO, PPA, and TSA for the IEEE 85-bus distribution system with four capacitors. 


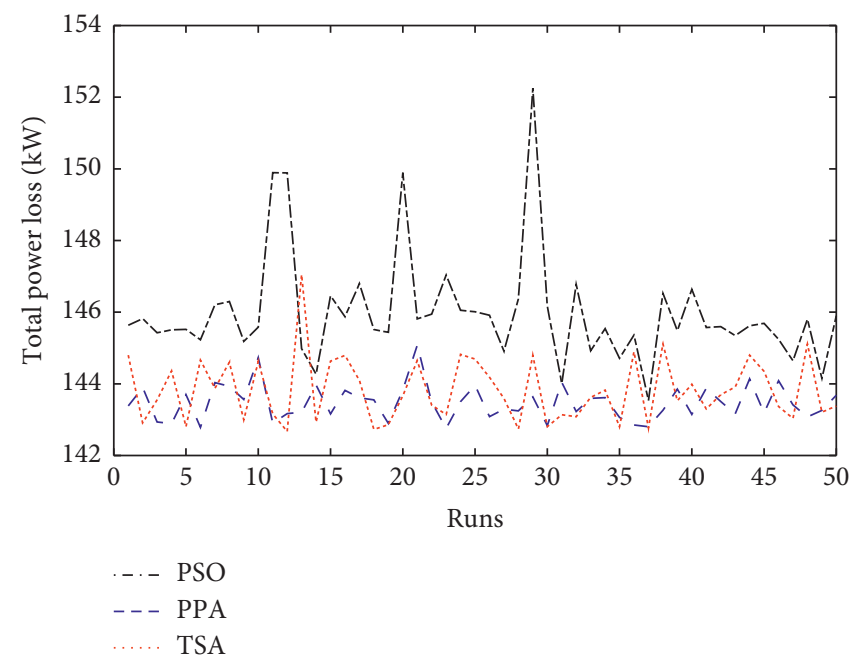

Figure 23: TAPL of 50 runs obtained by PSO, PPA, and TSA for the IEEE 85-bus distribution system with four capacitors.

TABLE 16: Result comparison for the IEEE 85-bus distribution system with four capacitors.

\begin{tabular}{|c|c|c|c|c|c|c|}
\hline Method & Capacitor site & Size (kVAr) & Total kVAr & Total loss $(\mathrm{kW})$ & Npo & Niter \\
\hline MINP [24] & $2,8,29,58$ & $300,700,900,500$ & 2400 & 159.87 & - & - \\
\hline PSO & $9,11,34,75$ & $468,113,352,197$ & $1130+1377=2507$ & 143.534 & 30 & 100 \\
\hline PPA & $9,30,49,67$ & $481,206,184,255$ & $1126+1377=2503$ & 142.753 & 30 & 100 \\
\hline TSA & $8,12,34,68$ & $406,173,320,223$ & $1122+1377=2499$ & 142.672 & 30 & 100 \\
\hline
\end{tabular}

TABLE 17: The improvement level of TSA over PPA and PSO for the four test systems.

\begin{tabular}{|c|c|c|c|c|c|c|}
\hline System & Case & Method & $p$ & $Z$ & $W$ & Improvement level \\
\hline \multirow{4}{*}{ IEEE 15 -bus system } & \multirow{2}{*}{1} & PSO & $<0.00001$ & -4.233 & 199 & Significant \\
\hline & & PPA & $<0.00001$ & -5.15 & 104 & Significant \\
\hline & \multirow{2}{*}{2} & PSO & $<0.00001$ & -4.7446 & 146 & Significant \\
\hline & & PPA & $<0.00001$ & -5.0149 & 118 & Significant \\
\hline \multirow{4}{*}{ IEEE 33-bus system } & \multirow{2}{*}{1} & PSO & $<0.0232$ & -2.2733 & 402 & Significant \\
\hline & & PPA & 0.267 & -1.115 & 522 & Insignificant \\
\hline & \multirow[b]{2}{*}{2} & PSO & 0.01552 & -2.4181 & 387 & Significant \\
\hline & & PPA & 0.86502 & -0.1689 & 620 & Insignificant \\
\hline \multirow{4}{*}{ IEEE 69-bus system } & \multirow{2}{*}{1} & PSO & 0.07672 & -1.7714 & 454 & Insignificant \\
\hline & & PPA & 0.00018 & -3.7406 & 250 & Significant \\
\hline & \multirow{2}{*}{2} & PSO & 0.4965 & -0.6806 & 567 & Insignificant \\
\hline & & PPA & $<0.00001$ & -4.1171 & 211 & Significant \\
\hline \multirow{4}{*}{ IEEE 85 -bus system } & \multirow{2}{*}{1} & PSO & $<0.00001$ & -5.5651 & 61 & Significant \\
\hline & & PPA & 0.16452 & -1.3949 & 493 & Insignificant \\
\hline & \multirow{2}{*}{2} & PSO & $<0.00001$ & -5.8547 & 31 & Significant \\
\hline & & PPA & 0.0536 & -1.9258 & 438 & Insignificant \\
\hline
\end{tabular}

\section{Conclusion}

In this paper, reactive power compensation in radial distribution systems has been investigated in reducing total power loss. On the contrary to other previous studies, the study has used local compensation at each load for increasing power factor to 0.9 and then capacitors in distribution lines have been placed as other studies. The combination could help three applied methods including PSO, PPA, and TSA to find the lowest power loss for four radial distribution systems with $15,33,69$, and 85 buses with two and three capacitors for the first three systems and three and four capacitors for the last system. The comparisons among the three methods indicated that TSA was the best one with the lowest loss and many runs with less loss than the best runs of PPA and PSO. The comparisons with previous methods indicated that TSA was much more effective. The improvement of TSA over other previous methods is up to $19.4 \%$ and $6.2 \%$ for Case 1 and Case 2 of the IEEE 15 -bus distribution system, $0.41 \%$ and $0.5 \%$ for Case 1 and Case 2 of the IEEE 33-bus distribution system, 1.1\% and 7.9\% for Case 1 and Case 2 of the IEEE 69-bus distribution system, and $5.98 \%$ and $10.76 \%$ for Case 1 and Case 2 of the IEEE 85-bus distribution system. Although PSO was worse 
than PPA and TSA for approximately all study cases, its results were more effective than other methods, even much better than its modified version. The improvement of PSO over previous method was up to $18.97 \%$ and $4.23 \%$ for Case 1 and Case 2 of the IEEE 15-bus distribution system, $0.4 \%$ and $0.18 \%$ for Case 1 and Case 2 of the IEEE 33-bus distribution system, $1.03 \%$ and $7.73 \%$ for Case 1 and Case 2 of the IEEE 69-bus distribution system, and 5.39\% and $10.21 \%$ for Case 1 and Case 2 of the IEEE 85-bus distribution system. The values did not mean that PSO was more effective than other previous methods; however, PSO could reach the good results thanks to the proposed compensation method. As a result, it can conclude that TSA is one of the most powerful methods that can be used in radial distribution system and the combination of local compensation at each load and compensation in distribution lines is very useful in reducing power loss of distribution systems.

\section{Nomenclature}

\begin{tabular}{|c|c|}
\hline Ndl: & Number of distribution lines \\
\hline$Z_{l}:$ & Impedance of the $l$ th distribution line \\
\hline$N n:$ & $\begin{array}{l}\text { Number of nodes in the considered } \\
\text { distribution network }\end{array}$ \\
\hline $\mathrm{FF}_{x}$ : & Fitness value of the solution $x$ \\
\hline$S_{x}:$ & The $x$ th solution \\
\hline$S_{\text {Gbest }}:$ & The most effective solution in the population \\
\hline$\varepsilon_{1}, \varepsilon_{2}, \varepsilon_{3}$ & Random numbers within 0 and 1 \\
\hline$\varepsilon_{4}, \varepsilon_{5}:$ & \\
\hline$\gamma_{1}, \gamma_{2}, \gamma_{3}:$ & Random numbers within 0 and 1 \\
\hline$r d_{1}:$ & Random number within 0 and 1 \\
\hline$\Delta S_{1}, \Delta S_{x}:$ & $\begin{array}{l}\text { The jumping step of the first solution and the } \\
x \text { th solution }\end{array}$ \\
\hline$S_{1}, S_{1}^{\text {new }}:$ & $\begin{array}{l}\text { The first old solution and the first new solution } \\
\text { in population }\end{array}$ \\
\hline$S_{x}, S_{x}^{\text {new }}:$ & $\begin{array}{l}\text { The } x \text { th old solution and the } x \text { th new solution } \\
\text { in population }\end{array}$ \\
\hline$Q_{\text {Loadk: }}$ & Reactive power of load at the $k$ th node \\
\hline$\Delta U_{l}:$ & Voltage drop on the $l$ th distribution line \\
\hline$U^{\min }, U^{\max }:$ & Lower bound and upper bound of load voltage \\
\hline$U_{k}:$ & Voltage of the node $k$ \\
\hline Npo: & Size of population \\
\hline Niter: & Number of iterations \\
\hline Citer: & Current iteration. \\
\hline
\end{tabular}

\section{Data Availability}

The data of the studied systems in the paper were taken from $[29,32,34]$.

\section{Conflicts of Interest}

The authors declare that they have no conflicts of interest.

\section{Acknowledgments}

This research is funded by Vietnam National Foundation for Science and Technology Development (NAFOSTED) under Grant number 102.02-2020.07.

\section{References}

[1] M. H. Haque, "Capacitor placement in radial distribution systems for loss reduction," IEE Proceedings-Generation, Transmission and Distribution, vol. 146, no. 5, pp. 501-505, 2013.

[2] A. Jafari, H. Ganjeh Ganjehlou, T. Khalili, B. MohammadiIvatloo, A. Bidram, and P. Siano, "A two-loop hybrid method for optimal placement and scheduling of switched capacitors in distribution networks," IEEE Access, vol. 8, pp. 3889238906, 2020.

[3] R. Viral and D. K. Khatod, "An analytical approach for sizing and siting of DGs in balanced radial distribution networks for loss minimization," International Journal of Electrical Power \& Energy Systems, vol. 67, pp. 191-201, 2015.

[4] A. R. Abul'Wafa, "Optimal capacitor allocation in radial distribution systems for loss reduction: a two stage method," Electric Power Systems Research, vol. 95, pp. 168-174, 2013.

[5] K. Prakash and M. Sydulu, "Particle swarm optimization based capacitor placement on radial distribution systems," in Proceedings of the 2007 IEEE Power Engineering Society General Meeting, Tampa, FL, USA, 2007.

[6] M. D. Reddy and V. V. Reddy, "Capacitor placement using fuzzy and particle swarm optimization method for maximum annual savings," ARPN Journal of Engineering and Applied Sciences, vol. 3, no. 3, pp. 25-30, 2008.

[7] R. S. Bapu, C. Prakash, and S. M. Kannan, "Optimal capacitor allocation in 69-bus radial distribution system to improve annual cost savings for dynamic load," International Journal of Emerging Technology and Advanced Engineering, vol. 3, no. 3, pp. 834-840, 2013.

[8] E. S. Ali, S. M. Abd Elazim, and A. Y. Abdelaziz, "Improved harmony algorithm and power loss index for optimal locations and sizing of capacitors in radial distribution systems," International Journal of Electrical Power \& Energy Systems, vol. 80, pp. 252-263, 2016.

[9] A. A. El-Ela, A. M. Kinawy, M. T. Mouwafi, and R. A. ElSehiemy, "Optimal sitting and sizing of capacitors for voltage enhancement of distribution systems," in Proceedings of the 2015 50th International Universities Power Engineering Conference (UPEC), Stoke on Trent, UK, 2015.

[10] S. Kansal, V. Kumar, and B. Tyagi, "Hybrid approach for optimal placement of multiple DGs of multiple types in distribution networks," International Journal of Electrical Power \& Energy Systems, vol. 75, pp. 226-235, 2016.

[11] D. Q. Hung, N. Mithulananthan, and R. C. Bansal, "Multiple distributed generator placement in primary distribution networks for loss reduction," IEEE Transactions on Industrial Electronics, vol. 60, no. 4, pp. 1700-1708, 2013.

[12] A. Hamouda, N. Lakehal, and K. Zehar, "Heuristic method for reactive energy management in distribution feeders," Energy Conversion and Management, vol. 51, no. 3, pp. 518-523, 2010.

[13] A. Bayat and A. Bagheri, "Optimal active and reactive power allocation in distribution networks using a novel heuristic approach," Applied Energy, vol. 233-234, pp. 71-85, 2019.

[14] D. Q. Hung, N. Mithulananthan, and R. C. Bansal, "A combined practical approach for distribution system loss reduction," International Journal of Ambient Energy, vol. 36, no. 3, pp. 123-131, 2016.

[15] S. Kamel, A. Amin, A. Selim, and M. H. Ahmed, "Optimal placement of DG and capacitor in radial distribution systems considering load variation," in Proceedings of the 2019 International Conference on Computer, Control, Electrical, and Electronics Engineering (ICCCEEE), Khartoum, Sudan, 2019. 
[16] C.-S. Lee, H. V. H. Ayala, and L. d. S. Coelho, "Capacitor placement of distribution systems using particle swarm optimization approaches," International Journal of Electrical Power \& Energy Systems, vol. 64, pp. 839-851, 2015.

[17] N. Kanwar, P. Saini, N. Gupta, A. Swarnkar, and K. R. Niazi, "Genetic algorithm based method for capacitor placement using new sensitivity based approach," in Proceedings of the 2014 18th National Power Systems Conference (NPSC), Guwahati, India, 2014.

[18] G. G. Soma, "Optimal sizing and placement of capacitor banks in distribution networks using a genetic algorithm," Electricity, vol. 2, no. 2, pp. 187-204, 2021.

[19] M. D. Reddy, "Optimal capacitor placement using fuzzy and real coded genetic algorithm for maximum savings," Journal of Theoretical and Applied Information Technology, vol. 4, no. 3, pp. 219-226, 2008.

[20] D. Das, "Optimal placement of capacitors in radial distribution system using a fuzzy-GA method," International Journal of Electrical Power \& Energy Systems, vol. 30, no. 6, pp. 361-367, 2008.

[21] K. R. Murthy, M. R. Raju, and G. G. Rao, "Comparison between conventional, GA and PSO with respect to optimal capacitor placement in agricultural distribution system," in Proceedings of the 2010 Annual IEEE India Conference (INDICON), Kolkata, India, 2010.

[22] S. Lohia, O. P. Mahela, and S. R. Ola, "Optimal capacitor placement in distribution system using genetic algorithm," in Proceedings of the 2016 IEEE 7th Power India International Conference (PIICON), Bikaner, India, 2016.

[23] M. Mahdavian, M. H. Kafi, A. Movahedi, and M. Janghorbani, "Improve performance in electrical power distribution system by optimal capacitor placement using genetic algorithm," in Proceedings of the 2017 14th International Conference on Electrical Engineering/Electronics, Computer, Telecommunications and Information Technology (ECTI-CON), Phuket, Thailand, 2017.

[24] S. Nojavan, M. Jalali, and K. Zare, "Optimal allocation of capacitors in radial/mesh distribution systems using mixed integer nonlinear programming approach," Electric Power Systems Research, vol. 107, pp. 119-124, 2014.

[25] A. M. Imran and M. Kowsalya, "Optimal distributed generation and capacitor placement in power distribution networks for power loss minimization," in Proceedings of the 2014 International Conference on Advances in Electrical Engineering (ICAEE), Vellore, India, 2014.

[26] K. R. Devabalaji, K. Ravi, and D. P. Kothari, "Optimal location and sizing of capacitor placement in radial distribution system using bacterial foraging optimization algorithm," International Journal of Electrical Power \& Energy Systems, vol. 71, pp. 383-390, 2015.

[27] Y. M. Shuaib, M. S. Kalavathi, and C. C. A. Rajan, "Optimal capacitor placement in radial distribution system using gravitational search algorithm," International Journal of Electrical Power \& Energy Systems, vol. 64, pp. 384-397, 2015.

[28] S. Sultana and P. K. Roy, "Optimal capacitor placement in radial distribution systems using teaching learning based optimization," International Journal of Electrical Power \& Energy Systems, vol. 54, pp. 387-398, 2014.

[29] A. Khodabakhshian and M. H. Andishgar, "Simultaneous placement and sizing of DGs and shunt capacitors in distribution systems by using IMDE algorithm," International Journal of Electrical Power \& Energy Systems, vol. 82, pp. 599-607, 2016.
[30] A. Y. Abdelaziz, E. S. Ali, and S. M. Abd Elazim, "Flower pollination algorithm and loss sensitivity factors for optimal sizing and placement of capacitors in radial distribution systems," International Journal of Electrical Power \& Energy Systems, vol. 78, pp. 207-214, 2016.

[31] V. Tamilselvan, T. Jayabarathi, T. Raghunathan, and X.-S. Yang, "Optimal capacitor placement in radial distribution systems using flower pollination algorithm," Alexandria Engineering Journal, vol. 57, no. 4, pp. 2775-2786, 2018.

[32] D. B. Prakash and C. Lakshminarayana, "Optimal siting of capacitors in radial distribution network using whale optimization algorithm," Alexandria Engineering Journal, vol. 56, no. 4, pp. 499-509, 2017.

[33] K. R. Devabalaji, T. Yuvaraj, and K. Ravi, "An efficient method for solving the optimal sitting and sizing problem of capacitor banks based on cuckoo search algorithm," Ain Shams Engineering Journal, vol. 9, no. 4, pp. 589-597, 2008.

[34] E. Mohamed, A.-A. A. Mohamed, and Y. Mitani, "MSA for optimal reconfiguration and capacitor allocation in radial-ring distribution networks," International Journal of Interactive Multimedia and Artificial Intelligence, vol. 5, no. 1, pp. 107-122, 2018.

[35] A. Rajendran and K. Narayanan, "Optimal multiple installation of DG and capacitor for energy loss reduction and loadability enhancement in the radial distribution network using the hybrid WIPSO-GSA algorithm," International Journal of Ambient Energy, vol. 41, no. 2, pp. 129-141, 2020.

[36] Y. Wu and Q. Song, "Improved particle swarm optimization algorithm in power system network reconfiguration," Mathematical Problems in Engineering, vol. 2021, Article ID 5574501, 10 pages, 2021.

[37] A.-A. A. Mohamed, S. A. Hassan, A. M. Hemeida, S. Alkhalaf, M. M. M. Mahmoud, and A. M. Baha Eldin, "Parasitism-predation algorithm (PPA): a novel approach for feature selection," Ain Shams Engineering Journal, vol. 11, no. 2, pp. 293-308, 2020.

[38] S. Kaur, L. K. Awasthi, A. L. Sangal, and G. Dhiman, “Tunicate swarm algorithm: a new bio-inspired based metaheuristic paradigm for global optimization," Engineering Applications of Artificial Intelligence, vol. 90, Article ID 103541, 2020.

[39] J.-H. Teng and C.-Y. Chang, "Backward/forward sweep-based harmonic analysis method for distribution systems," IEEE Transactions on Power Delivery, vol. 22, no. 3, pp. 1665-1672, 2007.

[40] http://www.socscistatistics.com/tests/signedranks/Default2. aspx.

[41] J. Derrac, S. García, D. Molina, and F. Herrera, "A practical tutorial on the use of nonparametric statistical tests as a methodology for comparing evolutionary and swarm intelligence algorithms," Swarm and Evolutionary Computation, vol. 1, no. 1, pp. 3-18, 2011.

[42] T. T. Nguyen, V. Q. Nguyen, and V. D. Le, "Improved firefly algorithm: a novel method for optimal operation of thermal generating units," Complexity, vol. 2018, Article ID 7267593, 23 pages, 2018. 